\title{
ARTICLE OPEN \\ Disruption of Dhcr7 and Insig1/2 in cholesterol metabolism causes defects in bone formation and homeostasis through primary cilium formation
}

\author{
Akiko Suzuki $^{1,2}$, Kenichi Ogata $\mathbb{D}^{1,2}$, Hiroki Yoshioka $\mathbb{D}^{1,2}$, Junbo Shim ${ }^{1,2}$, Christopher A. Wassif $^{3}$, Forbes D. Porter ${ }^{3}$ and Junichi Iwata ${ }^{1,2,4,5}$
}

Human linkage studies suggest that craniofacial deformities result from either genetic mutations related to cholesterol metabolism or high-cholesterol maternal diets. However, little is known about the precise roles of intracellular cholesterol metabolism in the development of craniofacial bones, the majority of which are formed through intramembranous ossification. Here, we show that an altered cholesterol metabolic status results in abnormal osteogenesis through dysregulation of primary cilium formation during bone formation. We found that cholesterol metabolic aberrations, induced through disruption of either Dhcr7 (which encodes an enzyme involved in cholesterol synthesis) or Insig1 and Insig2 (which provide a negative feedback mechanism for cholesterol biosynthesis), result in osteoblast differentiation abnormalities. Notably, the primary cilia responsible for sensing extracellular cues were altered in number and length through dysregulated ciliary vesicle fusion in Dhcr7 and Insig $1 / 2$ mutant osteoblasts. As a consequence, WNT/ $\beta$-catenin and hedgehog signaling activities were altered through dysregulated primary cilium formation. Strikingly, the normalization of defective cholesterol metabolism by simvastatin, a drug used in the treatment of cholesterol metabolic aberrations, rescued the abnormalities in both ciliogenesis and osteogenesis in vitro and in vivo. Thus, our results indicate that proper intracellular cholesterol status is crucial for primary cilium formation during skull formation and homeostasis.

Bone Research (2020)8:1

; https://doi.org/10.1038/s41413-019-0078-3

\section{INTRODUCTION}

Cellular cholesterol amounts are regulated by multiple steps of biosynthesis and feedback mechanisms. ${ }^{1}$ Infants with cholesterol metabolism aberrations have malformations in the craniofacial region. ${ }^{2-5}$ For example, mutations in genes involved in cholesterol synthesis (DHCR7, SC5D, and DHCR24) have been found in patients with Smith-Lemli-Opitz Syndrome (SLOS) lathosterolosis, and desmosterolosis, who display craniofacial bone abnormalities. ${ }^{1}$ In addition, high-cholesterol diets during pregnancy are known to be a risk factor for birth defects, including craniofacial bone abnormalities. ${ }^{6,7}$ Despite these findings, it remains unclear how cholesterol metabolism contributes to craniofacial bone formation, particularly intramembranous ossification.

The 7-dehydrocholesterol reductase (DHCR7) catalyzes the final step of cholesterol biosynthesis; ${ }^{8}$ mutations in DHCR7 cause cholesterol deficiency and an excess of cholesterol precursors, resulting in craniofacial deformities (e.g., microcephaly, cleft palate, craniosynostosis, and micrognathia), intellectual disability, and behavioral problems in humans. ${ }^{9,10} \mathrm{Dhcr}^{-1-}$ mice show a suckling defect, weight less, immature lungs, distended bladders, and variable craniofacial abnormalities. ${ }^{11}$ The molecular mechanism of craniofacial anomalies in these conditions is still elusive. The insulin-induced genes 1 and 2 (INSIG1 and INSIG2) are endoplasmic reticulum (ER) retention proteins that play roles in both the regulation of the activity of the 3-hydroxy-3-methylglutarylcoenzyme A (HMG-CoA) reductase and the translocation of the sterol regulatory element-binding protein (SREBP) to the nucleus for gene regulation. ${ }^{12}$ Mice deficient for Insig1 and Insig2 (Insig $1^{-1}$ ;Insig2 ${ }^{-1-}$ mice), which are negative regulators of cholesterol biosynthesis, ${ }^{13}$ show high-cholesterol levels in craniofacial tissues and display craniofacial deformities such as midfacial cleft, cleft palate, calvarial deformities and micrognathia, while mice deficient for either Insig1 or Insig2 are normal. ${ }^{3,12}$ These craniofacial deformities are rescued by the normalization of cholesterol levels in Insig1/2 null mice, ${ }^{3}$ however, it remains elusive how high-cholesterol levels cause craniofacial deformities and which cells are responsible for the craniofacial anomalies seen in Insig1/2 null mice.

Primary cilia, microtubule-based organelles that function in sensory and signaling pathways, are enriched with cholesterol-rich microdomains (known as lipid rafts) that recruit or retain receptors and ciliary membrane proteins. ${ }^{14}$ An association between lipid rafts and ciliary membrane proteins has been suggested in other organisms, including vertebrate photoreceptors, ${ }^{15}$ Chlamydomonas reinhardtii, ${ }^{16}$ mammalian spermatozoa, ${ }^{17}$ and Leishmania major. ${ }^{18}$ Defects of primary cilia cause various deformities, including craniofacial abnormalities (altogether known as ciliopathies, a group of genetic syndromes associated with defects in

\footnotetext{
${ }^{1}$ Department of Diagnostic \& Biomedical Sciences, The University of Texas Health Science Center at Houston, School of Dentistry, Houston, TX, USA; ${ }^{2}$ Center for Craniofacial Research, The University of Texas Health Science Center at Houston, School of Dentistry, Houston, TX, USA; ${ }^{3}$ Division of Translational Medicine, Eunice Kennedy Shriver National

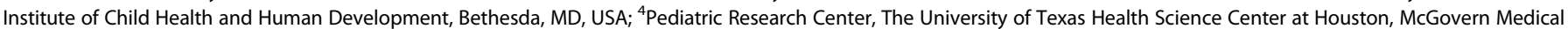
School, Houston, TX, USA and ${ }^{5}$ MD Anderson Cancer Center UTHealth Graduate School of Biomedical Sciences, Houston, TX, USA

Correspondence: Junichi Iwata (Junichi.Iwata@uth.tmc.edu)
}

Received: 17 April 2019 Revised: 20 August 2019 Accepted: 26 August 2019

Published online: 02 January 2020 
primary cilia). ${ }^{19,20}$ The broad-spectrum phenotypes in $\mathrm{Dhcr} 7^{-1-}$ mice and individuals with $\operatorname{SLOS}^{11}$ (e.g., presenting with craniofacial anomalies such as craniosynostosis, hypertelorism, and cleft palate, as well as immature lungs and enlarged bladders) are similar to those seen in ciliopathies. The phenotypic similarity between ciliopathies and cholesterol synthesis defects suggests that cholesterol metabolism (level and function of mature cholesterol and cholesterol intermediates) can regulate bone development through modulation of primary cilium formation and function. While over the past decade the underlying mechanism of ciliopathies has focused on the inner structures of primary cilia such as intraflagellar transport (IFT) and kinesin (KIF) proteins, ${ }^{21}$ little is known about the role of the surface membrane characteristics of primary cilia in ciliogenesis.

In this study, we investigated the link between cholesterol metabolic aberrations and craniofacial bone abnormalities by employing both loss-of-function and gain-of-function mouse models: mice with a deletion of Dhcr7 and mice with a deletion of Insig1/2, respectively. To identify the bone abnormalities with either low or high-cholesterol levels, we carried out microCT, skeletal staining, and histological analyses in these mice. Our study aims to elucidate how Dhcr7 and Insig1/2 regulate bone formation.

\section{RESULTS}

Dhcr7 deficiency increases osteogenesis

Dhcr ${ }^{-1-}$ knockout (KO) mice presented microcephaly, accelerated bone formation, and thicker calvaria bones at birth with complete penetrance, and died within 1 day after birth (Fig. 1a-c and Supplementary Fig. S1). The accelerated bone formation resulted in immature suture fusion after culturing calvaria explants for 3 days (Supplementary Fig. S1d). To examine the cellular mechanism of how cholesterol metabolic aberrations cause accelerated bone formation in $D h c r 7^{-1-}$ mice, we carried out biological analyses, namely BrdU incorporation assays and Ki67 immunohistochemistry for cell proliferation, TUNEL assays for apoptosis, and von Kossa staining for mineralization, and immunoblotting for type I collagen for osteogenic differentiation. We found that osteogenic differentiation, but not cell proliferation and apoptosis, was increased in Dhcr ${ }^{-1-}$ frontal bones (Fig. 1d, e and Supplementary Fig. S2). Next, to determine the regulatory mechanism of osteogenic differentiation, we performed quantitative RT-PCR (qRT-PCR) analyses for osteogenic factors (Runx2, Alp, Col1a1, Col1a2, Bglap, Sparc, Sp7, and Spp1) using frontal bones from $\mathrm{DhCr}^{-1-}$ mice at embryonic day (E) E14.5, E15.5, E16.5, and postnatal day (P) P0 (Fig. 1f and Supplementary Fig. S3). Col1a1 gene expression was significantly and consistently upregulated in Dhcr ${ }^{-1-}$ frontal bones compared to controls at E14.5-P0. Expression of RUNX2, COL1A1 and SP7 (aka Osterix) was increased, and the area with these positive signals was expanded compared to controls and correlated with increased expression of these genes in $D h c r 7^{-1-}$ frontal bones (Fig. If, g). Next, we evaluated the effect of loss of Dhcr7 on osteogenic differentiation using cultured osteoblasts from PO frontal bones. The Dhcr $7^{-/-}$ osteoblast characteristics (no proliferation defect but accelerated osteogenic differentiation through upregulated Col1a1 expression) were well conserved in cultured primary osteoblasts (Fig. 1h and Supplementary Fig. S3). Taken together, our results indicate that a failure in cholesterol synthesis causes accelerated osteogenesis through upregulated Colla1 expression.

Insig1/2 deficiency suppresses osteogenesis

To determine the tissue-specific contribution of high-cholesterol status to craniofacial deformities, we employed Insig1/2 conditional knockout (cKO) mice ${ }^{12}$ in cranial neural crest (CNC) cells ${ }^{22}$, which give origin to the majority of craniofacial bones. ${ }^{23}$ Insig1/2 cKO mice were viable, but they exhibited very thin frontal bones at birth and later in life (Fig. 2a, b), while the other craniofacial structures derived from CNC cells in these mice were intact. To examine the cellular mechanism of how high-cholesterol amount results in decreased bone formation in Insig1/2 cKO mice, we carried out biological analyses, as performed in $D h c r 7^{-1-}$ mice. We found that osteogenic differentiation, but not cell proliferation and apoptosis, was decreased in Insig1/2 cKO frontal bones (Supplementary Fig. S4). We also found that Col1a1 gene expression was significantly and consistently downregulated in frontal bones of Insig1/2 CKO mice at E14.5, E16.5 and PO (Fig. 2c), with protein expression correlating with gene expression (Fig. 2d). Expression of COL1A1 and SP7, but not RUNX2, was decreased, which was correlated with the expressions of these genes in Insig1/2 cKO frontal bones (Fig. 2e). We further evaluated the effect of loss of Insig1/2 on osteogenic differentiation using cultured osteoblasts from Insig1/2 cKO mice (Fig. 2f and Supplementary Fig. S4). The Insig 1/2 cKO osteoblast characteristics (no proliferation defect but reduced osteogenic differentiation through decreased Col1a1 expression) were well conserved in cultured primary osteoblasts. Since COL1 expression was decreased in Insig1/2 cKO mice, the abnormalities may resemble osteogenesis imperfecta, which is a congenital bone disorder characterized by thinner and fragile bones that affects 6-7 in 100 000 individuals worldwide. ${ }^{24}$ Altogether, our results indicate that either too much or too little cholesterol causes calvarial bone abnormalities through dysregulation of Col1a1 expression.

Ciliogenesis is altered in either Dhcr7- or Insig1/2-deficient mice As cholesterol is abundant in cellular membranes, we carefully investigated cellular membranous structures and found that there were fewer and shorter primary cilia in $\mathrm{Dhcr} 7^{-1-}$ osteoblasts compared to controls (Fig. 3a-c). Ciliogenesis starts with the interaction of the basal body (mother centriole) with primary ciliary vesicles (CVs), which can be labeled with $R A B 11,{ }^{25}$ and then the axoneme grows within the ciliary membrane while fusing with secondary CVs, which can be labeled with RAB8. ${ }^{26}$ The elongated primary cilium eventually fuses with the plasma membrane, allowing the distal part of the cilium to interact with the extracellular milieu. ${ }^{19,27}$ To track ciliogenesis in Dhcr $7^{-1-}$ osteoblasts, CVs were immunostained with anti-RAB11 and anti-RAB8 antibodies. We found that RAB11-positive primary CVs accumulated in the cells and that RAB8-positive secondary CVs failed to gather at sites of cilium formation in Dhcr $7^{-1-}$ osteoblasts (Fig. 3d, e).

On the other hand, Insig1/2 cKO osteoblasts displayed multiple and longer primary cilia compared to controls (Fig. 4a-c), and RAB8-positive CVs were abnormally fused with each other (Fig. 4d). The osteogenic and primary cilium phenotypes in Insig1/2 cKO osteoblasts were recapitulated with transfection of an adenovirus-Cre system (Supplementary Fig. S5). In addition, multiple basal bodies were detected, consistent with the presence of multiple primary cilia in Insig1/2 cKO osteoblasts (Fig. 4e and Supplementary Figs. S5 and S6). We then hypothesized that gene expression of molecules involved in the formation of the pericentriolar material (PCM) complex (i.e., Atf5, Aurka, Bbs4, Cdk5rap2, Cep152, Cep192, Cpap, Lck, Nin, Pcm1, Pcnt, Plk1, Plk4, Sass6, Stil, Tube1, Tubg1, Tunks, and Tunks2), which is important for proper centriole formation, was altered in Insig1/2 cKO osteoblasts. Previous studies indicate that overexpression of PIk1, Plk4, Stil and Sass6 induces the formation of multiple centrioles. ${ }^{28-35}$ Among them, by conducting a bioinformatics promoter analysis we found that the Plk4 promoter contained two potential sterol regulatory elements (SREs) for the SRE-binding protein (SREBP) binding. To access the binding experimentally, we carried out chromatin immunoprecipitation (ChIP) assays for the sites [site 1; GTGGAGAGT (-244 bp to $-252 \mathrm{bp}$ ) and site 2; TCACTCAGC (-1295 bp to $-1303 \mathrm{bp})]$ in Insig1/2 CKO and control osteoblasts and found increased binding of SREBP1 and SREBP2 to the SREs in Insig1/2 cKO osteoblasts (Fig. 4f). Indeed, expression of the Plk4 gene was significantly and specifically upregulated in Insig1/2 cKO 


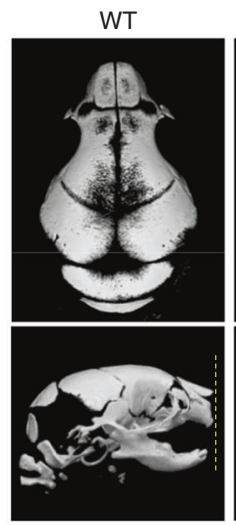

C
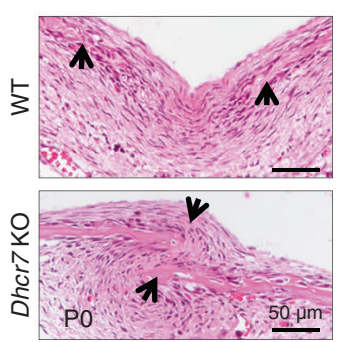

b

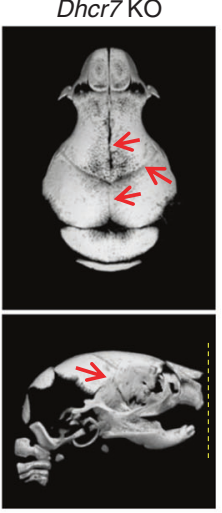

d

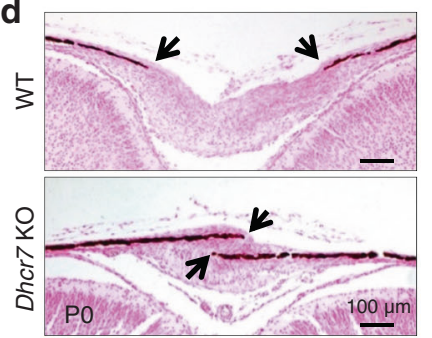

Dhcr7 KO
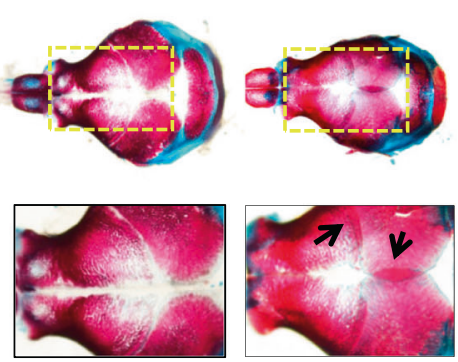

f

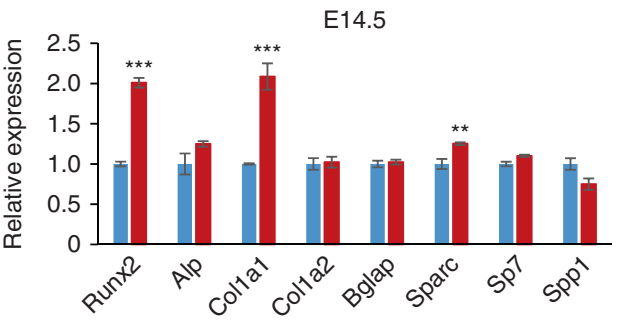

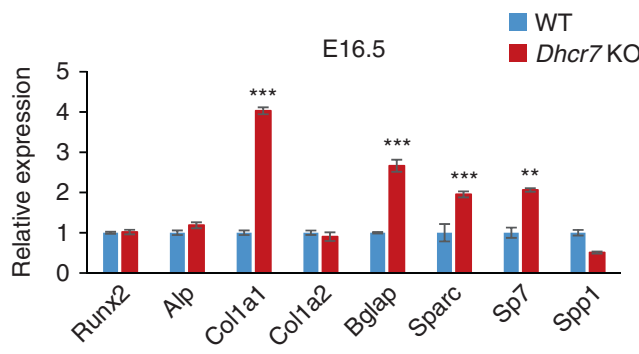

g

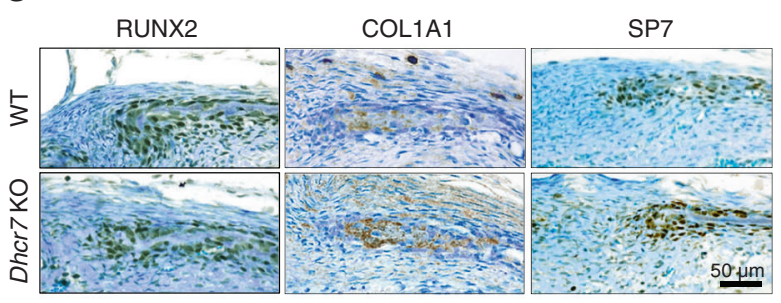

h

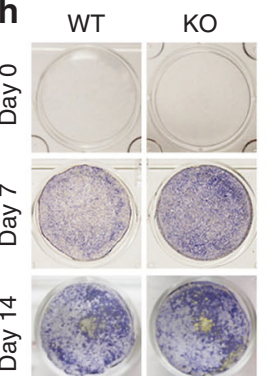

e WT KO

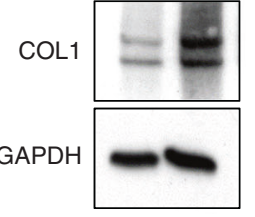

WT

Fig. 1 Loss of Dhcr7 accelerates osteogenesis. a MicroCT images of the calvaria-top view (upper panels) and side view (lower panels) - of newborn wild-type (WT) control and Dhcr $7^{-1-}$ knockout (KO) mice. Red arrows indicate early closure of the metopic, coronal, and sagittal sutures. Yellow dotted lines in the lower panels indicate the tips of premaxillae. b Skeletal staining of skulls from newborn WT control and KO mice. Yellow boxed areas are enlarged in the lower images. Black arrows in KO images indicate overlapping of frontal and parietal bones at the coronal suture and left and right parietal bones at the sagittal suture. c Hematoxylin and Eosin (H\&E) staining of the sagittal sutures of newborn WT and KO mice. Arrows indicate the osteogenic front. Scale bar, $50 \mu \mathrm{m}$. $\mathbf{d}$ von Kossa staining of the sagittal sutures of newborn WT and KO mice. Arrows indicate the osteogenic front. Scale bar, $100 \mu \mathrm{m}$. e Immunoblotting for type I collagen (COL1) in P0 calvaria of WT and KO mice. GADPH was used as loading control. f Quantitative RT-PCR of the indicated osteogenic genes at E14.5 (left) and E16.5 (right) in WT (blue bars) and KO (red bars) mice. $n=6$ per genotype per stage. ${ }^{* *} P<0.01{ }^{* * *} P<0.001$. g Immunohistochemistry analysis for RUNX2, COL1A1 and SP7 (Osterix) in newborn WT and KO mice. Nuclei were counterstained with $0.04 \%$ methylene blue. Scale bar, $50 \mu \mathrm{m}$. $\mathbf{h}$ Alkaline phosphatase (left) and Alizarin Red (right) staining of osteoblasts isolated from newborn WT and KO calvaria after induction of osteogenic differentiation at Day 0, 7, and 14.

osteoblasts compared to controls (Fig. $4 \mathrm{~g}$ ), while the expression of other PCM-related genes was not altered (Supplementary Fig. S6b). We further confirmed that PLK4 protein expression was increased in the mutant osteoblasts compared to controls (Fig. 4h). Taken together, our results indicate that Dhcr7 and Insig1/2 play an important role in primary cilium formation.
WNT/ $\beta$-catenin and hedgehog signaling pathways are downstream signaling cascades responsible for the bone phenotype of Dhcr7- and Insig1/2-deficient mice

We examined how the ciliary phenotypes resulted in osteogenic abnormalities. The primary cilia coordinate with multiple signaling pathways such as hedgehog $(\mathrm{HH})$ and WNT during 

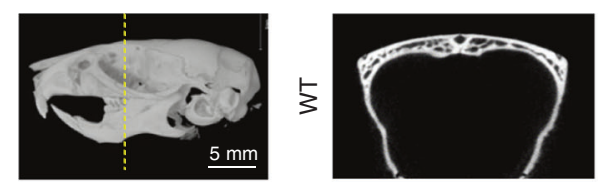

0
$\frac{1}{0}$
$\frac{1}{5}$
$\frac{.}{9}$
$\frac{5}{5}$

C

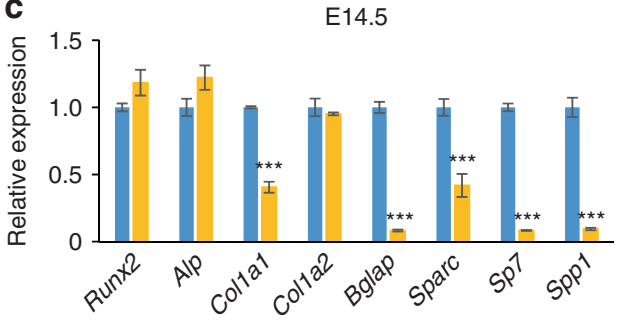

PO

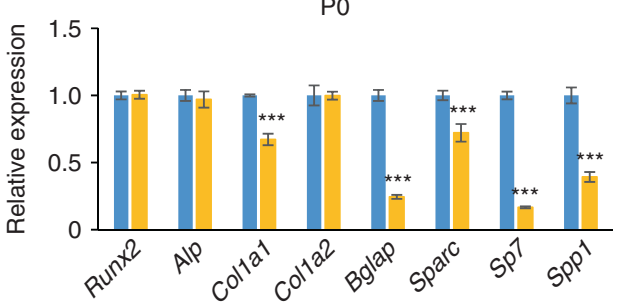

e

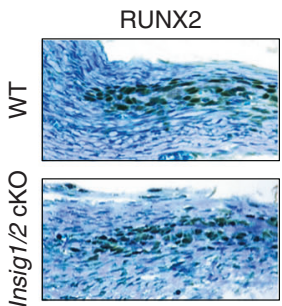

COL1A1

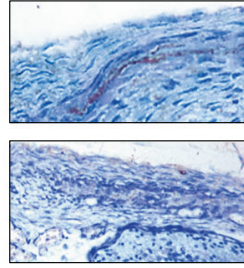

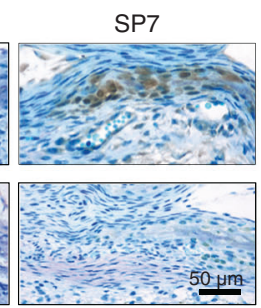

b

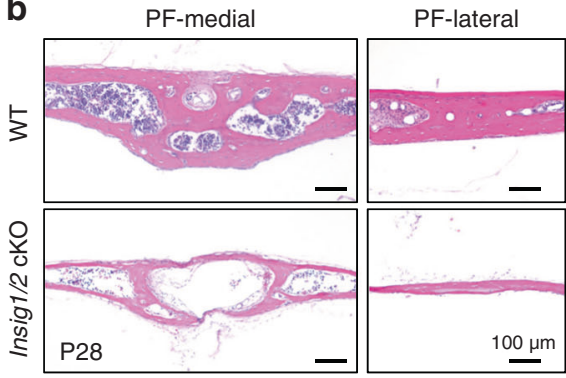

E16.5

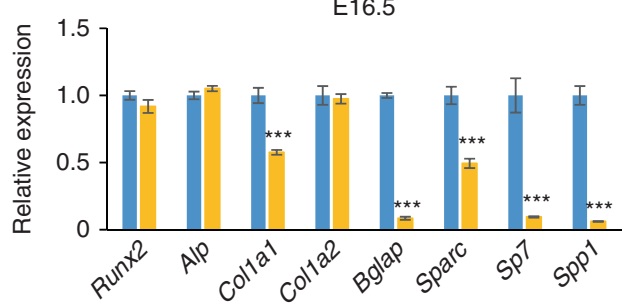

WT

Insig 1/2 cKO

d

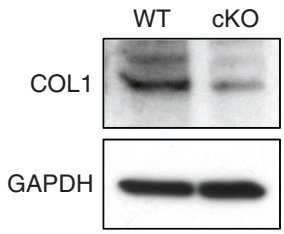

f

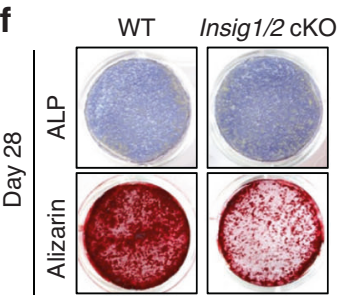

Fig. 2 Excessive cholesterol synthesis decreased bone formation in Insig1/2 cKO mice. a MicroCT images of the frontal bones (a slice at the level of the distal end of the 3rd molar, indicated by the yellow dotted line) of WT and Insig1/2 conditional KO (cKO) mice at P35. b H\&E staining of the medial and lateral regions of the posterior frontal bones (PF) from P28 WT and Insig $1 / 2$ cKO mice. Scale bars, $100 \mu \mathrm{m}$. c Quantitative RT-PCR for the indicated osteogenic genes in WT (blue bars) and Insig1/2 cKO (yellow bars) mice at E14.5, E16.5, and P0. $n=6$ per genotype per stage. ${ }^{* *} P<0.001$. d Immunoblotting for COL1 in newborn WT and Insig1/2 CKO mice. GADPH was used as loading control. e Immunohistochemical analysis for RUNX2, COL1A1 and SP7 (Osterix) in newborn WT and Insig1/2 cKO mice. Nuclei were counterstained with $0.04 \%$ methylene blue. Scale bar, $50 \mu \mathrm{m}$. f Alkaline phosphatase (top) and Alizarin Red (bottom) staining of osteoblasts isolated from the frontal bones of newborn WT and Insig1/2 cKO after induction of osteogenic differentiation at Day 28.

osteogenesis. $^{36-38}$ To investigate $\mathrm{HH}$ signaling in $\mathrm{Dhcr}^{-1-}$ calvaria, we analyzed Gli1 and Ptch1 levels by GRT-PCR, which are readout gene expressions for $\mathrm{HH}$ signaling activity. ${ }^{39-43} \mathrm{We}$ found that expression of these genes was significantly downregulated in $\mathrm{DhCr}^{-/-}$calvaria (Fig. 5a). Furthermore, we employed Gli1-LaCZ reporter mice to evaluate the $\mathrm{HH}$ signaling activity in vivo. We found that $\mathrm{HH}$ signaling was compromised in the calvaria of $D h c r 7^{-1-}$ mice compared to controls (Fig. 5b). To further confirm the reduced $\mathrm{HH}$ signaling activity in the calvaria of $D h c r 7^{-1-}$ mice, we performed cell fractionation and the consequent immunoblotting analyses for GLI1. Previous studies indicate that in the absence of $\mathrm{HH}$ ligands, GLI1 is truncated and degraded by the proteasome in the cytoplasm, while GLI1 translocates into the nucleus in the presence of $\mathrm{HH}$ ligands by escaping from degradation. ${ }^{44,45}$ As expected, full-length GLI1 was decreased in the nuclear fraction from $D h c r 7^{-1-}$ osteoblasts (Fig. 5c).
Next, we investigated the $\mathrm{HH}$ signaling activity in Insig1/2 cKO mice. We carried out qRT-PCR for Gli1 and Ptch1 in Insig1/2 cKO calvaria and found that expression of these genes was significantly upregulated in Insig1/2 CKO mice (Fig. 5d). Furthermore, we performed LacZ staining for Gli1 expression in Insig1/2 cKO;Gli1LacZ and control mice. As expected, Gli1-LacZ expression was increased in the calvaria of Insig1/2 cKO;Gli1-LacZ mice compared to that of control mice (Fig. 5e). Next, to examine whether Col1a1 expression was regulated through $\mathrm{HH}$ signaling, we conducted promoter analyses for GLI binding on the Col1a1 promoter and found that the Col1a1 promoter contained a putative GLI-binding site (GGCCACGCA; -68 bp to $-60 \mathrm{bp}$ ) (Supplementary Fig. S7a). ChIP assays validated that GLI binding to the Col1a1 promoter region was correlated with the activity of $\mathrm{HH}$ signaling in osteoblasts from $\mathrm{Dhcr}^{-1-}$ and Insig1/2 cKO mice (Fig. 5f, g).

Thus, $\mathrm{HH}$ signaling activity was correlated with the primary cilium phenotype of $\mathrm{Dhcr}^{-1-}$ and Insig1/2 cKO mice. However, 

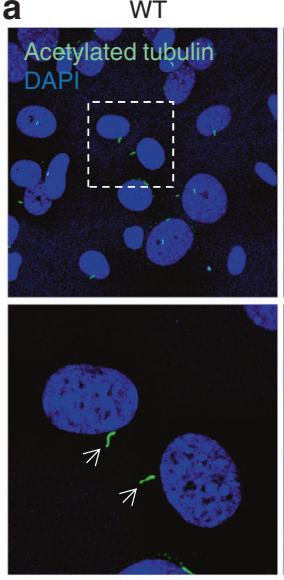

d

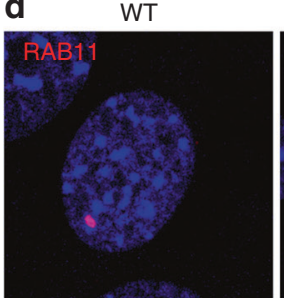

Dhcr7 KO

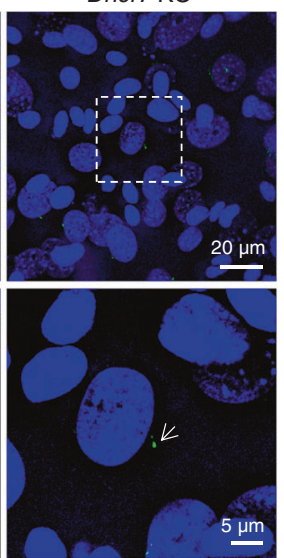

Dhcr7 KO

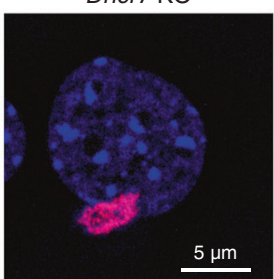

b
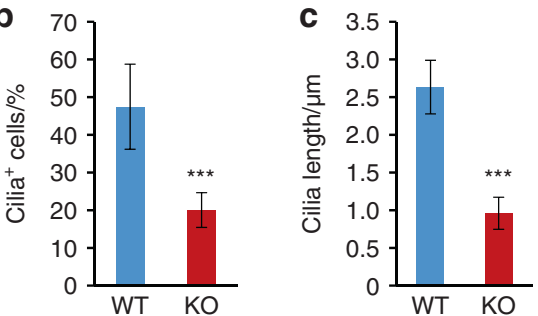

e
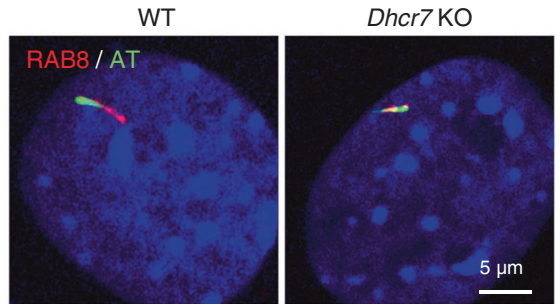

Fig. 3 Impaired cholesterol synthesis results in defective ciliogenesis in Dhcr7 KO osteoblasts. a Immunocytochemistry (IC) analyses of primary cilia in osteoblasts from wild-type (WT) control and Dhcr7 knockout (KO) mice. Primary cilia were stained with anti-acetylated tubulin antibody (green), and nuclei were stained with DAPI (blue). Boxed areas in upper images are enlarged, and arrows indicate primary cilia. Scale bars: $20 \mu \mathrm{m}$ in the upper images and $5 \mu \mathrm{m}$ in the lower images. Arrows indicate primary cilia. b Percentage of cells with primary cilia in osteoblasts from WT (blue bar) and Dhcr7 KO (red bar) mice. More than 200 cells were randomly analyzed in three independent experiments. ${ }^{* * *} P<0.001$. c Quantification of the length of primary cilia in osteoblasts from WT (blue bar) and Dhcr7 KO (red bar) mice. More than 200 cells were randomly analyzed in three independent experiments. ${ }^{* *} P<0.001$. d IC analyses for RAB11 (red) in WT and Dhcr7 KO osteoblasts. Nuclei were stained with DAPI (blue). Scale bar, 5 mm. e IC analyses for RAB8 (red) and acetylated tubulin (AT; green) in WT and Dhcr7 KO osteoblasts. Nuclei were stained with DAPI (blue). Scale bar, $5 \mu \mathrm{m}$.

previous studies show that upregulated and downregulated $\mathrm{HH}$ signaling cause defective and normal craniofacial bone formation, respectively. ${ }^{46-48}$ This suggests that altered $\mathrm{HH}$ signaling was not responsible for the bone phenotypes in $\mathrm{Dhcr} 7^{-1-}$ and Insig1/2 cKO mice.

We, therefore, investigated another candidate cilium-mediated signaling that regulates intramembranous ossification, WNT/ $\beta$-catenin signaling, ${ }^{49}$ in $D h c r 7^{-1}$ and Insig1/2 cKO mice. Recent studies indicate that primary cilia negatively regulate $W N T / \beta$-catenin signaling. ${ }^{37,50,51}$ To investigate the activity of WNT/ $\beta$-catenin signaling pathway, we conducted quantitative RT-PCR for Axin2, a readout of WNT/ $\beta$-catenin signaling activity, and found that its expression was upregulated and downregulated in $\mathrm{Dhcr}^{-1-}$ and Insig1/2 cKO osteoblasts, respectively (Fig. 6a, b and Supplementary Fig. S8). To validate the relationship between WNT/ $\beta$-catenin signaling and cholesterol metabolism, we treated primary osteoblasts from $\mathrm{Dhcr}^{-1-}$ and Insig1/2 cKO mice with either WNT3A or lithium chloride $(\mathrm{LiCl})$, a known WNT/ $\beta$-catenin signaling activator. While both WNT3A and $\mathrm{LiCl}$ induced Axin2 expression five-fold in wild-type controls, Axin2 expression was upregulated and downregulated in $D h c r 7^{-1-}$ and Insig 1/2 cKO osteoblasts, respectively (Fig. $6 c$, d). We confirmed that in mice with Topgal reporter for the WNT/ $\beta$-catenin signaling activity, its activity was increased at osteogenic front of the calvaria in $D h c r 7^{-1}$ mice compared to control mice (Fig. $6 \mathrm{6})$. Next, to examine whether Col1a1 expression was regulated through WNT/ $\beta$-catenin signaling, we conducted promoter analyses for $\beta$-catenin binding on the Col1a1 promoter and found four putative $\beta$-catenin binding sites (Supplementary Fig. S7b). To evaluate these predicted binding sites, we conducted ChIP assays for $\beta$-catenin binding to the Col1a1 promoter region, and found that $\beta$-catenin bound at binding sites 1 and 4 , and the binding was correlated with the activity of WNT/ $\beta$-catenin signaling in osteoblasts from $D h c r 7^{-1-}$ and Insig 1/2 cKO mice (Supplementary Fig. S9). Lastly, to test the functional significance of WNT/ $\beta$-catenin signaling, we generated and investigated compound mutant mice with a haploinsufficiency of Axin2 in the Dhcr7 mutant background and found that normalized WNT/ $\beta$-catenin signaling restored the accelerated bone formation in $D h c r 7^{-1-}$ mice (Fig. $6 \mathrm{f}-\mathrm{h}$ ). Altogether, these findings are well supported by the fact that WNT/ $\beta$-catenin signaling positively regulates osteogenesis. ${ }^{52}$

The bone phenotype in Dhcr7- and Insig1/2-deficient mice is restored by the normalization of aberrant cholesterol metabolism with simvastatin

To test whether abnormalities in primary cilium and bone formation can be restored by the normalization of aberrant cholesterol metabolism, we treated $\mathrm{Dhcr}^{-1-}$ and Insig1/2 cKO osteoblasts with simvastatin, which can normalize cholesterol metabolic aberrations by inhibiting the activity of the HMG-CoA reductase. Simvastatin treatment restored primary cilium formation in cultured osteoblasts from $\mathrm{Dhcr}^{-/-}$mice (Fig. 7a-c). In addition, the in vivo administration of simvastatin to $\mathrm{Dhcr} 7^{-/-}$ mice $\left(10 \mathrm{mg} \cdot \mathrm{kg}^{-1}\right.$ body weight per day, intraperitoneal injection to a pregnant mouse, E12.5-E18.5) could normalize the accelerated bone formation in newborn mice (Fig. 7d, e). We confirmed that expression of genes related to bone formation and WNT signaling was restored in $D h c r 7^{-1-}$ mice treated with simvastatin (Fig. 7f). These results indicate that cholesterol intermediates caused the bone defects in $D h c r 7^{-1-}$ mice. In Insig $1 / 2 \mathrm{cKO}$ mice, simvastatin treatment restored both the increased number and length of primary cilia (Fig. 8a, b) and the reduced bone formation in Insig1/2 CKO osteoblasts (Fig. 8c). Importantly, simvastatin treatment (10 $\mathrm{mg} \cdot \mathrm{kg}^{-1}$ body weight per day from P7 to P42) 
a
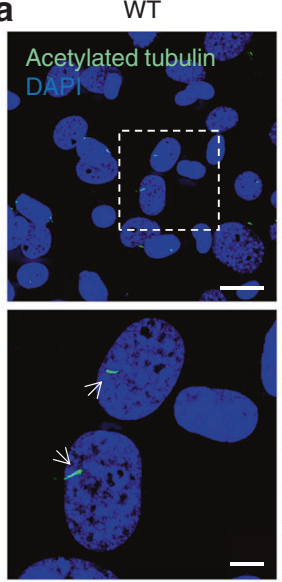

b

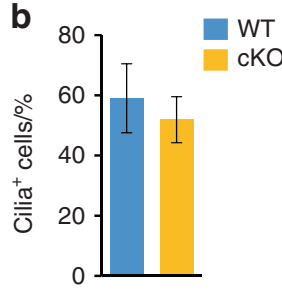

d
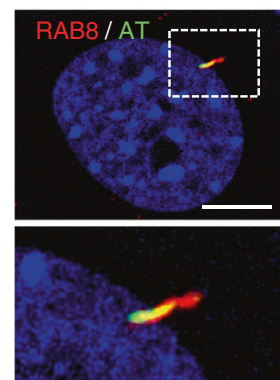

Insig 1/2 cKO
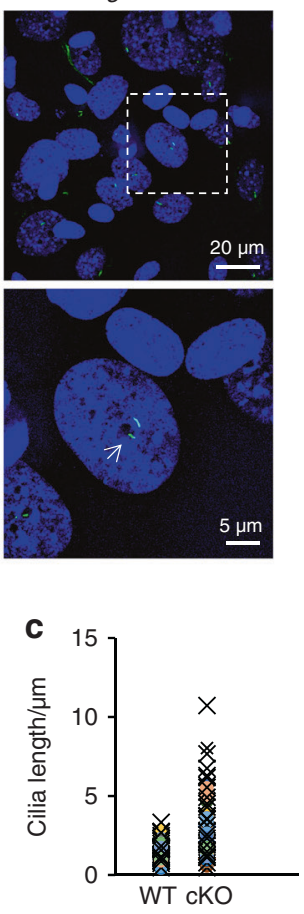

Insig 1/2 cKO

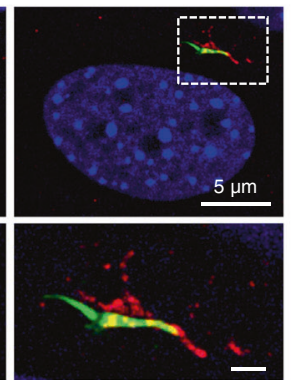

e
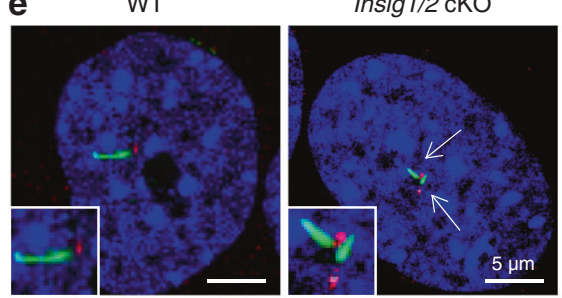

f

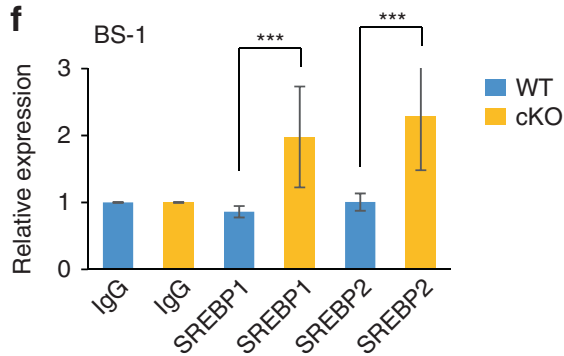

BS-2
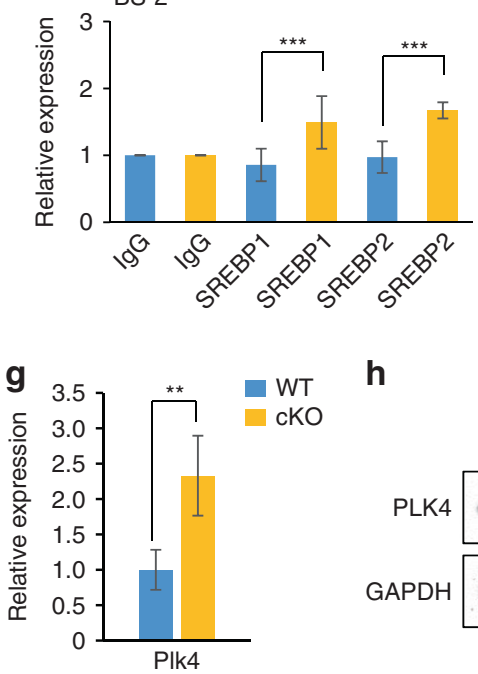

h

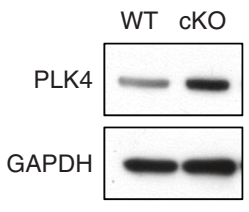

Fig. 4 Excessive cholesterol synthesis results in abnormal primary cilium formation. a IC analyses for anti-acetylated tubulin (AT; green) in WT and Insig1/2 conditional KO (cKO) osteoblasts. Nuclei were stained with DAPI (blue). Boxed areas in upper images are enlarged, and arrows indicate primary cilia. Scale bars: $20 \mu \mathrm{m}$ in the upper images and $5 \mu \mathrm{m}$ in the lower images. b Percentage of cells with primary cilia in osteoblasts from WT (blue bar) and Insig1/2 cKO (yellow bar) osteoblasts. More than 200 cells were randomly analyzed in three independent experiments. c Quantification of the length of primary cilia in osteoblasts from WT (left) and Insig $1 / 2$ cKO (right) mice. More than 200 cells were randomly analyzed in three independent experiments. ${ }^{* * *} P<0.001$. d IC for RAB8 (red) and AT (green) in WT and Insig1/2 cKO osteoblasts. Nuclei were stained with DAPI (blue). Boxed areas in upper images are enlarged. Scale bars, $5 \mu$ m. e IC analyses for $\gamma$-tubulin (red) and AT (green) in WT and Insig1/2 cKO osteoblasts. Nuclei were stained with DAPI (blue). Arrows indicate duplicated primary cilia and basal bodies. Primary cilia are enlarged in insets. Scale bars, $5 \mu \mathrm{m}$. $\mathbf{f}$ ChIP assays of IgG control and SREBP1 or SREBP2 for SRE (BS1 and BS2) of the Plk4 promoter region in WT control (blue bars) and Insig1/2 CKO (yellow bars) osteoblasts. $n=3$ per group. ${ }^{* *} P<0.001$. g Quantitative RT-PCR for Plk4 in WT (blue bar) and Insig1/2 cKO (yellow bar) osteoblasts. $n=6$ per group. ${ }^{* *} P<0.01$. $\mathbf{h}$ Immunoblotting for PLK4 in WT and Insig1/2 cKO osteoblasts. GAPDH was used as loading control.

improved the decreased bone formation in these mice (Fig. 8d). As expected, the expression of genes related to osteogenesis, cilia and WNT signaling was normalized in Insig1/2 cKO mice treated with simvastatin (Fig. 8e). Taken together, our results indicate that proper cholesterol metabolic status is crucial for normal primary cilium formation, which is responsible for osteogenesis in osteoblasts (Fig. 9).

\section{DISCUSSION}

Previous studies show that cholesterol metabolism is important for the formation and homeostasis of bone and cartilage during endochondral ossification. ${ }^{53,54}$ For example, mice with a deletion of Scap, a regulator for cholesterol biosynthesis, in either postcranial somatic lateral plate mesodermal cells $\left(\mathrm{Scap}^{\mathrm{F} / \mathrm{F}} ; \mathrm{Prx} 1\right.$ -
Cre mice) or chondrocytes (Scap ${ }^{F / F} ;$ Col2a1-Cre mice), display compromised chondrogenesis and disorganized growth plates, resulting in short limbs. ${ }^{54}$ By contrast, increased intracellular cholesterol in mice deficient for Insig1/2 in postcranial somatic lateral plate mesodermal cells (Insig $1^{\mathrm{F} / \mathrm{F}} ;$ Insig $2^{-1-} ; \operatorname{Prx} 1$-Cre mice) or chondrocytes (Insig $1^{\mathrm{F} / \mathrm{F}} ;$ Insig2 ${ }^{-1-} ;$ Col2a1-Cre mice) show defects in growth plate organization and chondrogenesis, also resulting in short limbs. ${ }^{54}$ Thus, either too much and too low of cholesterol results in short limbs through defective endochondral ossification. In this study, we aimed to study the role of cholesterol in intramembranous ossification using both gain-offunction (Insig 1/2 CKO mice) and loss-of-function (Dhcr $7^{-1-}$ mice) mouse models, and found that cholesterol metabolism plays a role in intramembranous ossification in a dose-dependent manner. These findings suggest that effects of abnormal 


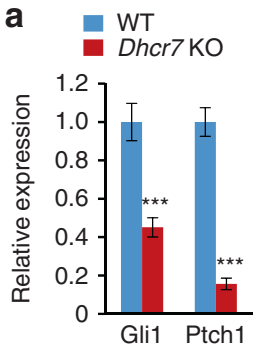

b

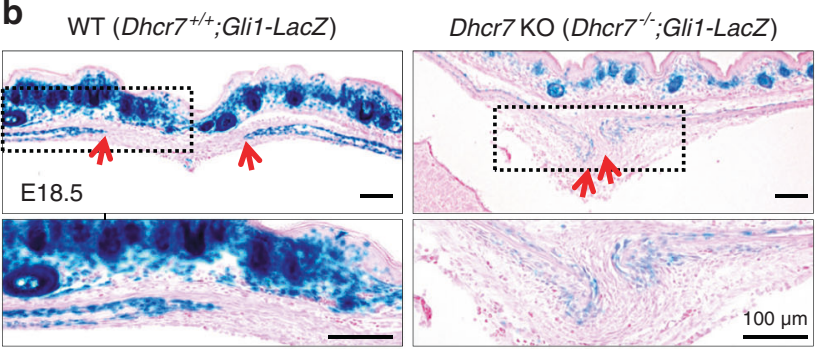

C

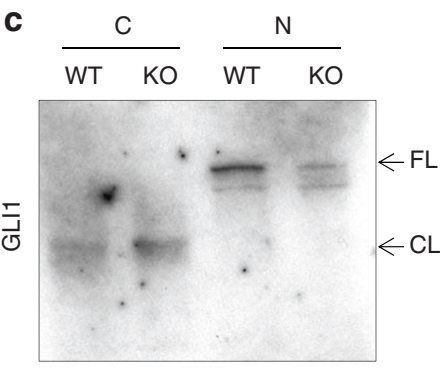

e

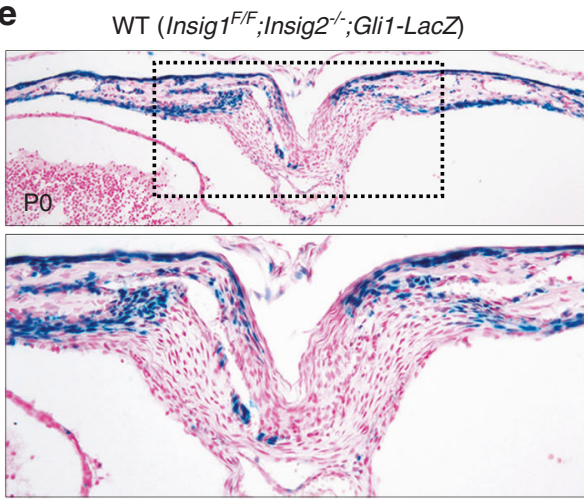

d

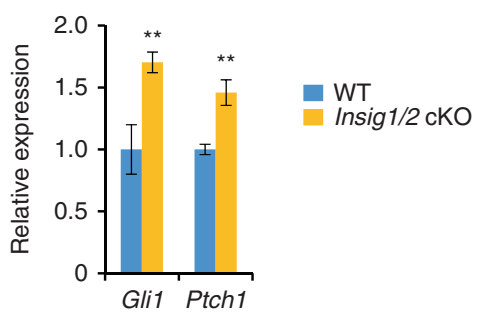

f Col1a1 ChIP in Drch7 GLI-BS

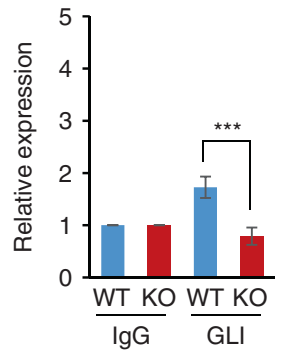

Insig1/2 cKO (Wnt1-Cre;Insig ${ }^{\mathrm{F} / \mathrm{F}} \cdot$ Insig $2^{-/} ;$Gli1-LacZ)

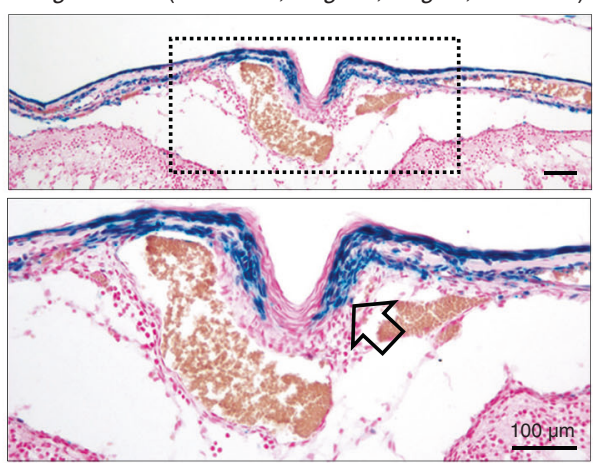

9 Col1a1 ChIP in Insig1/2

GLI-BS

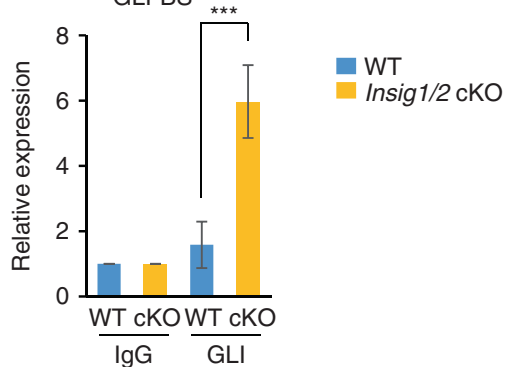

Fig. 5 Altered hedgehog signaling in calvaria from Dhcr7 and Insig1/2 mutant mice during craniofacial development. a Quantitative RT-PCR analyses for Gli1 and Ptch1 expression as a readout for HH signaling activity in newborn wild-type (WT) control (blue bars) and Dhcr7 knockout (KO; red bars) calvaria. $n=6$ per group. ${ }^{* *} P<0.001$. b $\beta$-galactosidase staining (blue) for sites of $\mathrm{HH}$ signaling activation in the frontal bones of E18.5 Dhcr7 ${ }^{+/+}$;Gli1-LacZ (WT) and Dhcr7 ${ }^{-1-}$;Gli1-LacZ (Dhcr7 KO) mice. Red arrows indicate osteogenic fronts; boxed areas are enlarged in lower images. Nuclei were stained with nuclear fast red. Scale bars, $100 \mu \mathrm{m}$. c Cell fractionation and subsequent immunoblotting analysis for GLI1, using cytosol (C) and nuclear (N) fractions from WT control and Dhcr7 KO osteoblasts. FL, full-length; CL, cleaved. d Quantitative RT-PCR analysis for Gli1 and Ptch1 in newborn WT control (blue bars) and Insig1/2 conditional KO (cKO; yellow bars) frontal bones. $n=6$ per group. ${ }^{* *} P<0.01$. e $\beta$-galactosidase staining (blue) for sites of $\mathrm{HH}$ signaling activation in the frontal bones of PO Insig $1^{F / F} ; \operatorname{Insig} 2^{-/-} ; G l i 1-L a c Z$ (WT) and

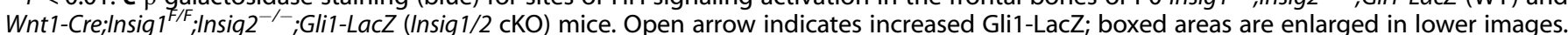
Nuclei were stained with nuclear fast red. Scale bars, $100 \mu \mathrm{m}$. f ChIP assays of IgG control and GLI-1 for the Col1a1 promoter region in Dhcr7 KO (red bars) and WT control (blue bars) osteoblasts. $n=3$ per group. ${ }^{* *} P<0.001$. g ChIP assays of IgG control and GLI- 1 for the Col1a 1 promoter region in Insig1/2 cKO (yellow bars) and WT control (blue bars) osteoblasts. $n=3$ per group. ${ }^{* * *} P<0.001$.

cholesterol metabolism differ between intramembranous and endochondral ossification.

7-DHC is a cholesterol intermediate as well as a precursor of vitamin $\mathrm{D} ; 7-\mathrm{DHC}$ is converted to pre-vitamin $\mathrm{D}_{3}$ by ultraviolet light on the skin and further converted to active vitamin $D_{3}$ in the liver and kidney. ${ }^{55}$ Previous studies indicate that suppression of DHCR7 activity results in upregulated vitamin $D_{3}$ levels in vivo after birth. ${ }^{56-58}$ While patients with SLOS show a high level of 
a

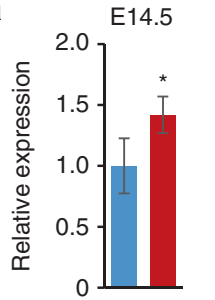

C

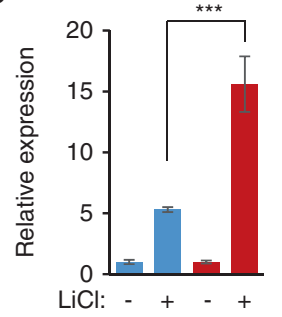

E16.5

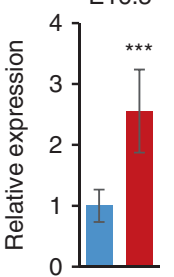

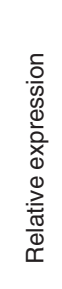

PO

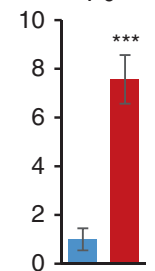

b

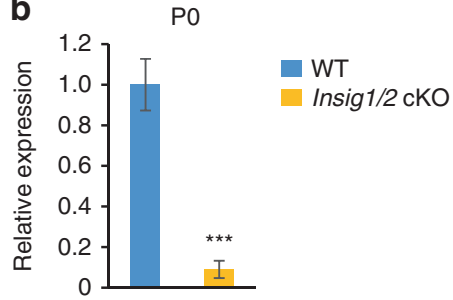

d

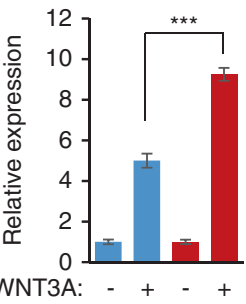

WT

Dhcr7 KO

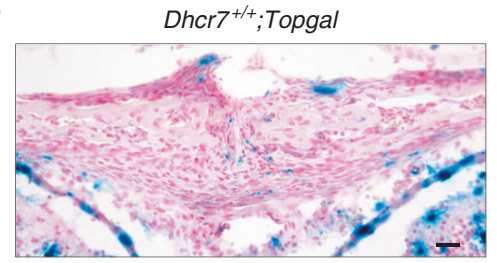

f
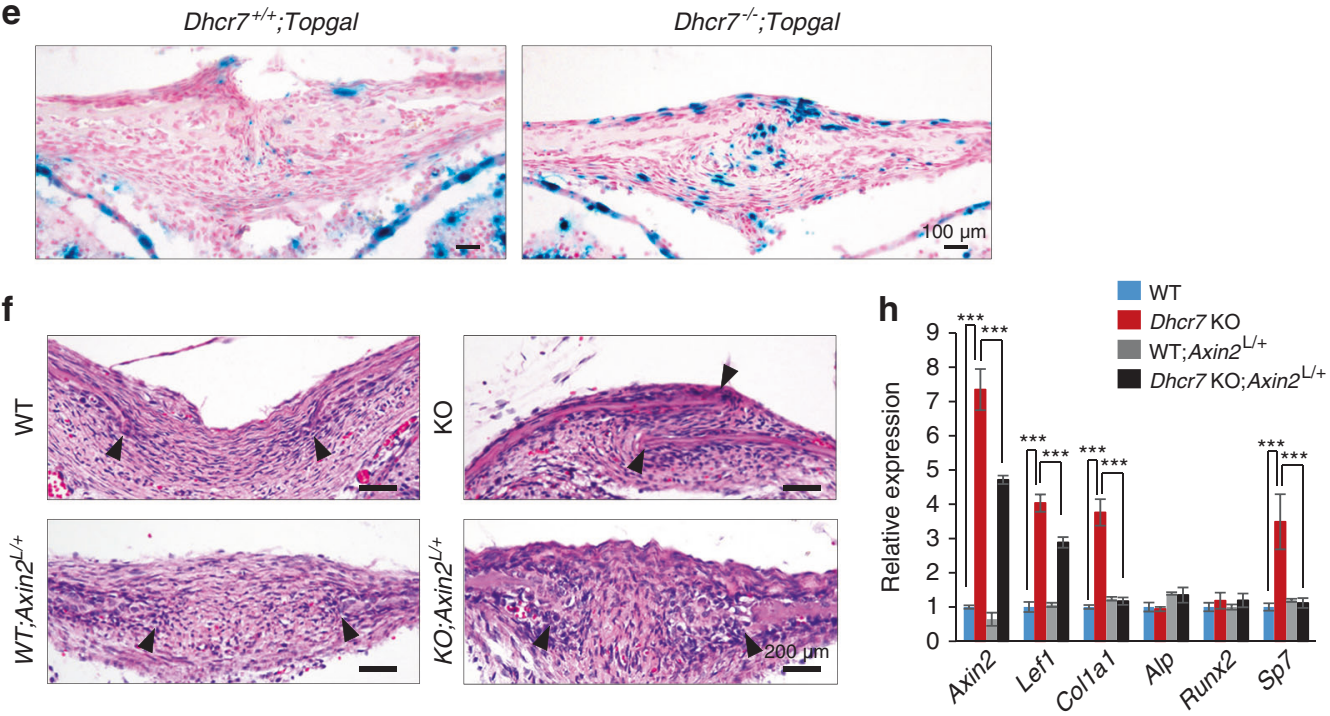

g

WT; Axin2 $^{L /+}$

Dhcr7 KO;Axin2 ${ }^{++}$

Dher7 KO;Axin2 ${ }^{L+}$

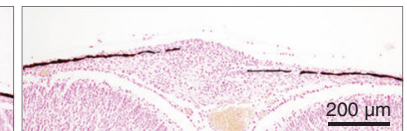

Fig. 6 Altered WNT/ $\beta$-catenin signaling in calvaria from Dhcr7 and Insig1/2 mutant mice during craniofacial development. a Quantitative RTPCR for Axin2 in calvaria from wild-type (WT; blue bars) control and Dhcr7 knockout (KO; red bars) mice at E14.5, E16.5, and P0 (newborn). $n=6$ per group. ${ }^{*} P<0.05 ;{ }^{* * *} p<0.001$. b Quantitative RT-PCR for Axin2 in calvaria from newborn WT (blue bar) and Insig1/2 conditional KO (cKO; yellow bar) mice. $n=6$ per group. ${ }^{* * *} P<0.001$. c Quantitative RT-PCR for Axin2 after treatment with LiCl (left panel) or WNT3A (right panel) in WT (blue bars) and Dhcr7 KO (red bars) osteoblasts. $n=6$ per group. ${ }^{* *} P<0.001$. d Quantitative RT-PCR for Axin2 after treatment with LiCl (left panel) or WNT3A (right panel) in WT (blue bars) and Insig1/2 cKO (yellow bars) osteoblasts. $n=6$ per group. ${ }^{* * *} P<0.001$. e $\beta$-galactosidase

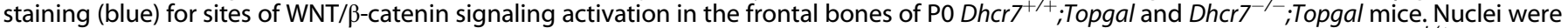
stained with nuclear fast red. Scale bars, $100 \mu \mathrm{m}$. f Hematoxylin and Eosin staining of the sagittal sutures of newborn WT, WT;Axin2 ${ }^{L /+}, D h c r 7 K O$ and Dhcr7 KO;Axin2 ${ }^{L /+}$ mice. Arrowheads indicate the osteogenic front. Accelerated bone formation of the sutures (frontal, coronal, and sagittal sutures) was normalized in newborn $D h c r 7 K O ; A x i n 2^{L /+}$ mice $(n=6 / 6)$. Scale bars, $100 \mu \mathrm{m}$. g Von Kossa staining of the sagittal sutures of newborn WT;Axin2 ${ }^{L /+}$, Dhcr7 KO and Dhcr7 KO;Axin $2^{L /+}$ mice. Scale bar, $200 \mu \mathrm{m}$. h Quantitative RT-PCR of the indicated genes in newborn WT (blue bars), Dhcr7 KO (red bars), WT;Axin2 ${ }^{L+}$ (gray bars) and Dhcr7KO;Axin2 ${ }^{L++}$ (black bars) mice. $n=6$ per group. ${ }^{* * *} P<0.001$.

vitamin $D_{3}$ compared with healthy individuals, calcium homeostasis is not affected. ${ }^{59}$ As vitamin $D_{3}$ biosynthesis is inactive during embryogenesis due to lack of exposure to ultraviolet light, and because only maternal 25 -hydroxyvitamin $D$ is a source of vitamin $D_{1}^{60,61}$ the effect of vitamin $D_{3}$ on bone development would be minimal. By contrast, while the maternal cholesterol supply partially restores the exogenous cholesterol supply in embryos, embryonic tissues still need to synthesize cholesterol through endogenous cholesterol synthesis pathways in each organ to compensate for the gap between the external supply and their needs. ${ }^{62-64}$ This could be a possible reason why cholesterol metabolic anomalies cause different degree/severity of malformations in different tissues in mice and humans.

Primary cilia transduce extracellular cues as a mechanical sensor, as well as a chemical sensor, for morphogens and growth factors. ${ }^{65,66}$ Therefore, both endochondral and intramembranous ossification are affected in mice with defective primary cilia. ${ }^{38}$ For example, mice deficient for Kif3a, a ciliary protein, in osteoblasts 
a
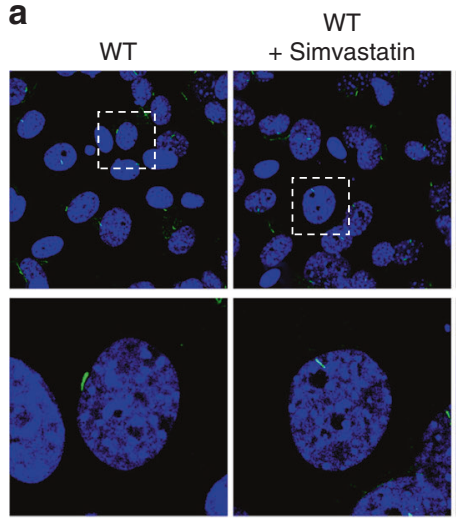

d

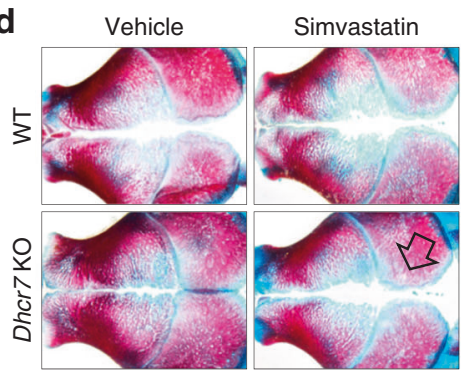

f

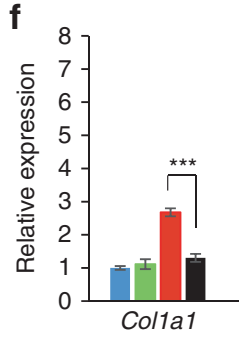

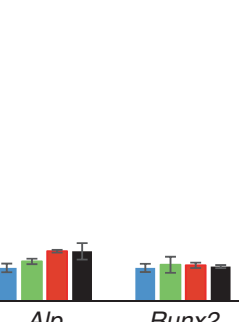

Runx2
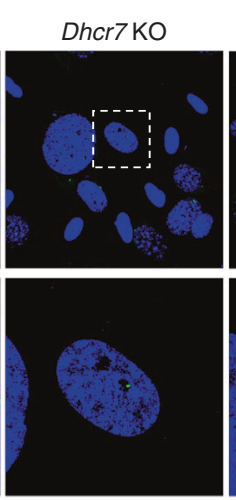

e
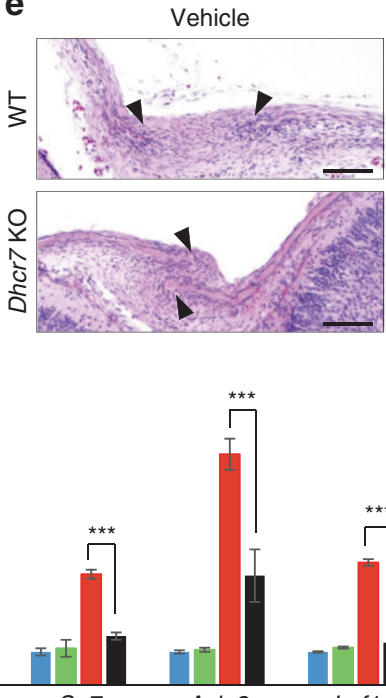

Sp7
Dhcr7 KO
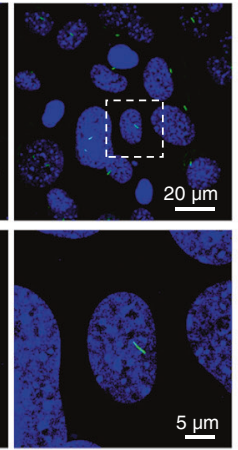
+ Simvastatin b

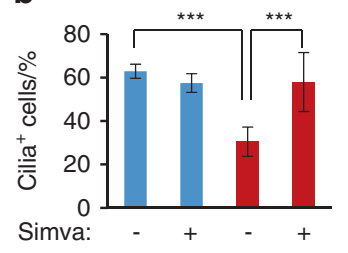

C

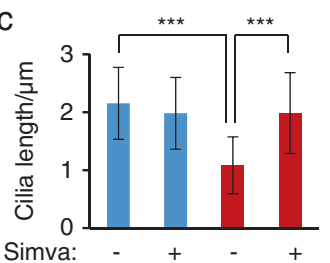

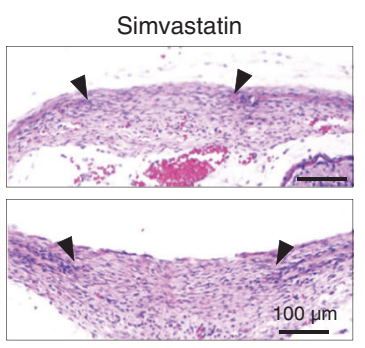

Fig. 7 Simvastatin rescues altered bone formation in Dhcr7 mutant mice. a Immunocytochemistry (IC) analyses of primary cilia in osteoblasts from wild-type (WT) control and Dhcr7 knockout (KO) mice after simvastatin treatment. Primary cilia were stained with anti-acetylated tubulin antibody (green), and nuclei were stained with DAPI (blue). Boxed areas in upper images are enlarged. Scale bars, $20 \mu \mathrm{m}$ in the upper images and $5 \mu \mathrm{m}$ in the lower images. b Percentage of cells with primary cilia in osteoblasts from WT (blue bars) and Dhcr7 KO (red bars) mice after treatment with simvastatin. $n=124$ per group. ${ }^{* * *} P<0.001$. c Quantification of the length of primary cilia in osteoblasts from WT (blue bars) and Dhcr7 (red bars) KO mice after simvastatin treatment. $n=124$ per group. ${ }^{* *} P<0.001$. d Skull staining after simvastatin treatment (10 mg $\mathrm{kg}^{-1}$ body weight, intraperitoneal injection to a pregnant mouse, E12.5-E18.5). The open arrow indicates rescued calvarial abnormalities. e Hematoxylin and Eosin staining of the sagittal sutures of newborn WT and Dhcr7 KO mice after simvastatin treatment. Arrowheads indicate the osteogenic front. Accelerated bone formation of the sutures (frontal, coronal, and sagittal sutures) was normalized in newborn Dhcr7 KO mice $(n=6 / 6)$. Scale bars, $100 \mu \mathrm{m}$. f Quantitative RT-PCR of the indicated genes in newborn WT (blue bars), WT treated with simvastatin (green bars), Dhcr7 KO (red bars), and Dhcr7 KO treated with simvastatin (black bars) mice. $n=6$ per group. ${ }^{* * *} P<0.001$.

display decreased bone formation and osteopenia. ${ }^{67}$ A deficiency for cilia-related protein SPEF2 results in osteoblast differentiation defects. $^{68}$ In this study, we found that $\mathrm{HH}$ and WNT/ $\beta$-catenin signaling is inverted in conditions of the primary cilia in $\mathrm{Dhcr} 7^{-/-}$ and Insig1/2 CKO mice during intramembranous ossification. While both WNT/ $\beta$-catenin and $\mathrm{HH}$ signaling pathways are essential for endochondral ossification as well as for the differentiation and maturation of cultured osteoblasts, ${ }^{36}$ previous mouse genetic studies suggest that WNT/ $\beta$-catenin signaling may be more dominant than $\mathrm{HH}$ signaling for osteogenesis in intramembranous ossification during skull formation.

In regard to $\mathrm{HH}$ signaling, previous studies show that $\mathrm{HH}$ signaling is compromised in Dhcr7 mutant mammalian models; for example, $\mathrm{HH}$ signaling is inhibited in $\mathrm{Dhcr} \mathrm{7}^{-1-}$ mouse embryonic fibroblasts (MEFs) ${ }^{69}$, as well as in mice deficient for Dhcr7 (Dhcr $7^{\Delta E \times 8 / \Delta E \times 8}$ mice). ${ }^{70,71}$ Our results show that the number of ciliated cells, as well as the length of cilia, is decreased in $\mathrm{Dhcr} 7^{-/-}$ osteoblasts, which is responsible for diminished $\mathrm{HH}$ signaling.
Previous studies indicate that $\mathrm{HH}$ signaling is not dominant in regulating intramembranous ossification; for example, mice with loss of Ihh ( $/ h^{-1-}$ mice), which is expressed at the osteogenic front in cranial bones, ${ }^{72,73}$ develop small but normal calvaria, ${ }^{47,48}$ and osteogenesis is not affected during intramembranous ossification. ${ }^{74}$ Mice deficient for Smo $\left(\mathrm{Smo}^{n / c} ;\right.$ Wnt1-Cre mice) display small but normal skulls in contrast to other severe craniofacial defects. ${ }^{75}$ In addition, mice deficient for Shh $\left(S h h^{n / c}\right.$; K14-Cre mice and $S h h^{F / F} ; K 14-C r e$ mice), which is specifically expressed in the epithelium in craniofacial regions, show normal skull formation while they exhibit cleft palate. 2,76,77 Thus, loss-offunction of $\mathrm{HH}$ signaling does not affect calvaria formation. By contrast, gain-of-function of $\mathrm{HH}$ signaling results in defective bone formation; for example, mice with an ectopic $\mathrm{HH}$ signaling activation in CNC cells $\left(\mathrm{Smo}^{+/ \mathrm{ML}^{2}}\right.$;Osr2-IrsCre) show cleft palate and osteogenesis defects such as the absence of the palatine processes of the premaxilla and maxilla and a short mandible with ossification defects. ${ }^{46}$ In addition, overexpression of Shh in the 

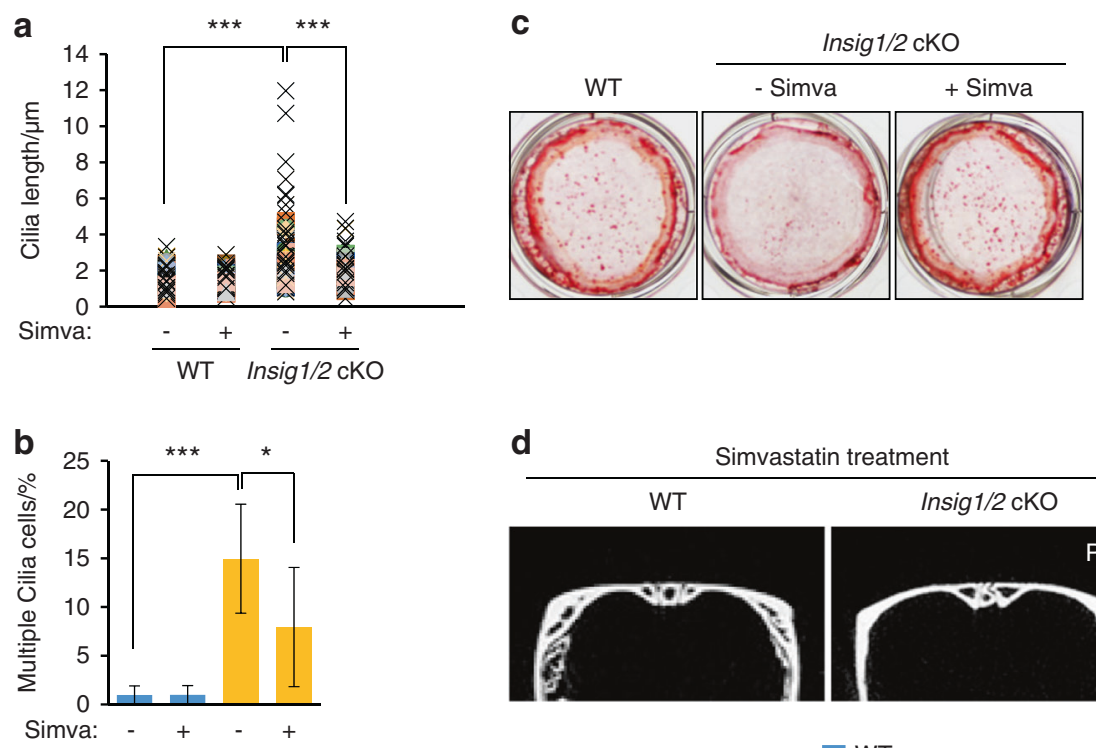

d
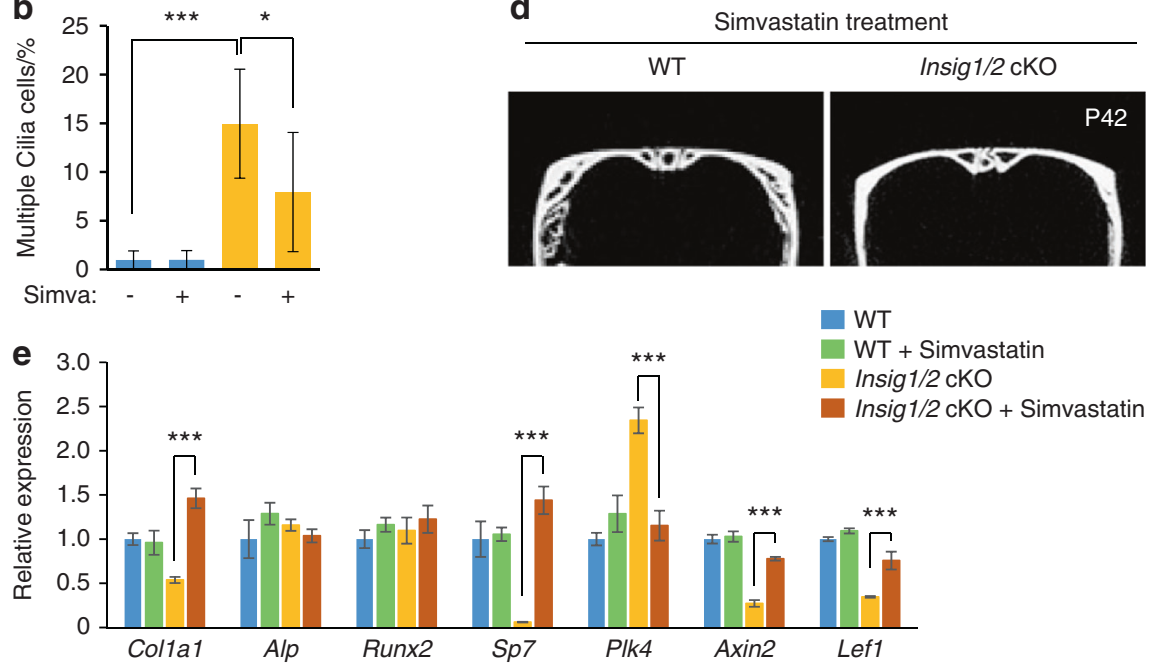

Fig. 8 Simvastatin rescues altered bone formation in Insig1/2 mutant mice. a Quantification of ciliary length in WT and Insig $1 / 2 \mathrm{cKO}$ osteoblasts with/without simvastatin treatment (Simva). $n=124$ per group. ${ }^{* *} P<0.001$. b Quantification of cells with multiple cilia with/without simvastatin treatment (Simva) in WT (blue bars) and Insig 1/2 CKO (yellow bars). $n=124$ per group. ${ }^{*} P<0.05 ;{ }^{* * *} p<0.001$. c Alizarin Red staining of osteoblasts isolated from newborn WT and Insig1/2 cKO frontal bones after induction of osteogenic differentiation with/without simvastatin treatment at Day 28. d MicroCT images from WT and Insig 1/2 cKO mice after simvastatin treatment (10 mg $\cdot \mathrm{kg}^{-1}$ body weight from P7 to P42). e Quantitative RT-PCR of the indicated genes in P42 WT (blue bars), WT treated with simvastatin (green bars), Insig1/2 cKO (yellow bars), and Insig1/2 cKO treated with simvastatin (brown bars) mice. $n=6$ per group. ${ }^{* *} P<0.001$.

epithelium (K14-Shh transgenic mice) results in craniofacial bone defects, cleft palate, short limbs, and polysyndactyly through the increased and expanded $\mathrm{SHH}$ signaling activation in the mesenchyme. ${ }^{78}$ Taken together, although $\mathrm{HH}$ signaling may contributes to intramembranous ossification at some degree, upregulation and downregulation of $\mathrm{HH}$ signaling in $\mathrm{Dhcr} 7^{-1-}$ and Insig 1/2 CKO mice is not in agreement with the hypothesis that $\mathrm{HH}$ is a factor responsible for cilia-mediated osteogenesis during intramembranous ossification.

As to WNT/ß-catenin signaling, previous studies show that it positively regulates osteogenesis; for example, constitutive active WNT/ $\beta$-catenin signaling in bones results in increased osteogenesis in mice, ${ }^{79-81}$ and WNT/ $\beta$-catenin signaling inhibition results in a failure of osteogenesis; for example, mice with inactivated $\beta$-catenin in mesoderm-derived osteoblast and chondrocyte progenitor cells (Catnby ${ }^{c / c}$;Dermo1-Cre mice) show drastically diminished osteogenesis and ectopic cartilage formation in both intramembranous and endochondral ossification. ${ }^{82} \mathrm{Kif3}^{-/-}$and Kif3 $a^{F / F}$;Wnt1-Cre mice as well as MEFs or embryonic stem (ES) cells from $\mathrm{Kif3}^{-1-}$, Ofd $1^{-1-}$ and Ift88 $8^{\text {orpk/orpk }}$ mice with no functional primary cilia show hyper-activated WNT/ $\beta$-catenin signaling, compared to ciliated control cells, ${ }^{83,84}$ as seen in $D h c r 7^{-1-}$ osteoblasts. Thus, WNT/ $\beta$-catenin signaling is well correlated with the osteogenic phenotype in $D h c r 7^{-1-}$ and Insig $1 / 2$ cKO mice during intramembranous ossification.

In summary, we demonstrated the biological significance of intracellular cholesterol metabolism in the regulation of osteogenesis and ciliogenesis using $D h c r 7^{-1-}$ and Insig1/2 cKO mice and derived cultured osteoblasts. Dhcr $7^{-1-}$ osteoblasts displayed fewer and shorter primary cilia compared to controls, while Insig1/2 cKO osteoblasts exhibited supernumerary and longer primary cilia compared to controls. Our work places a new focus on primary cilium formation regulated by cholesterol metabolism in the bone. The principles learned from this study promise to be fertile ground for future molecular genetic studies of craniofacial bone development, and may lead to the development of innovative preventive and therapeutic approaches for bone diseases and ciliopathies related to cholesterol metabolic aberrations.

\section{MATERIALS AND METHODS}

Animals

Dhcr ${ }^{-1-}$ mice ${ }^{85}$ were a gift from Dr. Forbes D. Porter (The Eunice Kennedy Shriver National Institute of Child Health and Human Development, National Institutes of Health, Bethesda, Maryland, USA). Insig $1^{F / F} ;$ Insig2 ${ }^{-/-}$(The Jackson Laboratory, \#005939) ${ }^{12}$ and Wnt1-Cre2 (The Jackson Laboratory, $\# 022501)^{22}$ mice were obtained from The Jackson Laboratory and crossed to generate Insig1/2 CKO mice. Gli1-LacZ mice (The Jackson Laboratory, $\# 008211)^{39}$ were obtained from The Jackson Laboratory and crossed with $\mathrm{Dhcr} 7^{+/-}$and Wnt1-Cre2; Insig $1^{\mathrm{F} /+} ; \operatorname{Insig} 2^{-/-}$mice in order to generate DhCr ${ }^{-/-}$;Gli1-LacZ, DhCr ${ }^{+/+} ;$Gli1-LacZ, Insig1/2 CKO;Gli1-LacZ, and Insig ${ }^{\text {F/F }}$;Insig2 ${ }^{-1-}$;Gli1-LacZ mice. Topgal (The Jackson Laboratory, \#004623) ${ }^{86}$ and Axin2 ${ }^{\text {LacZ/+ }}$ (The Jackson Laboratory, \#009120) ${ }^{87}$ mice were obtained from The Jackson Laboratory and crossed with $D h c r 7^{-1-}$ mouse line to generate 


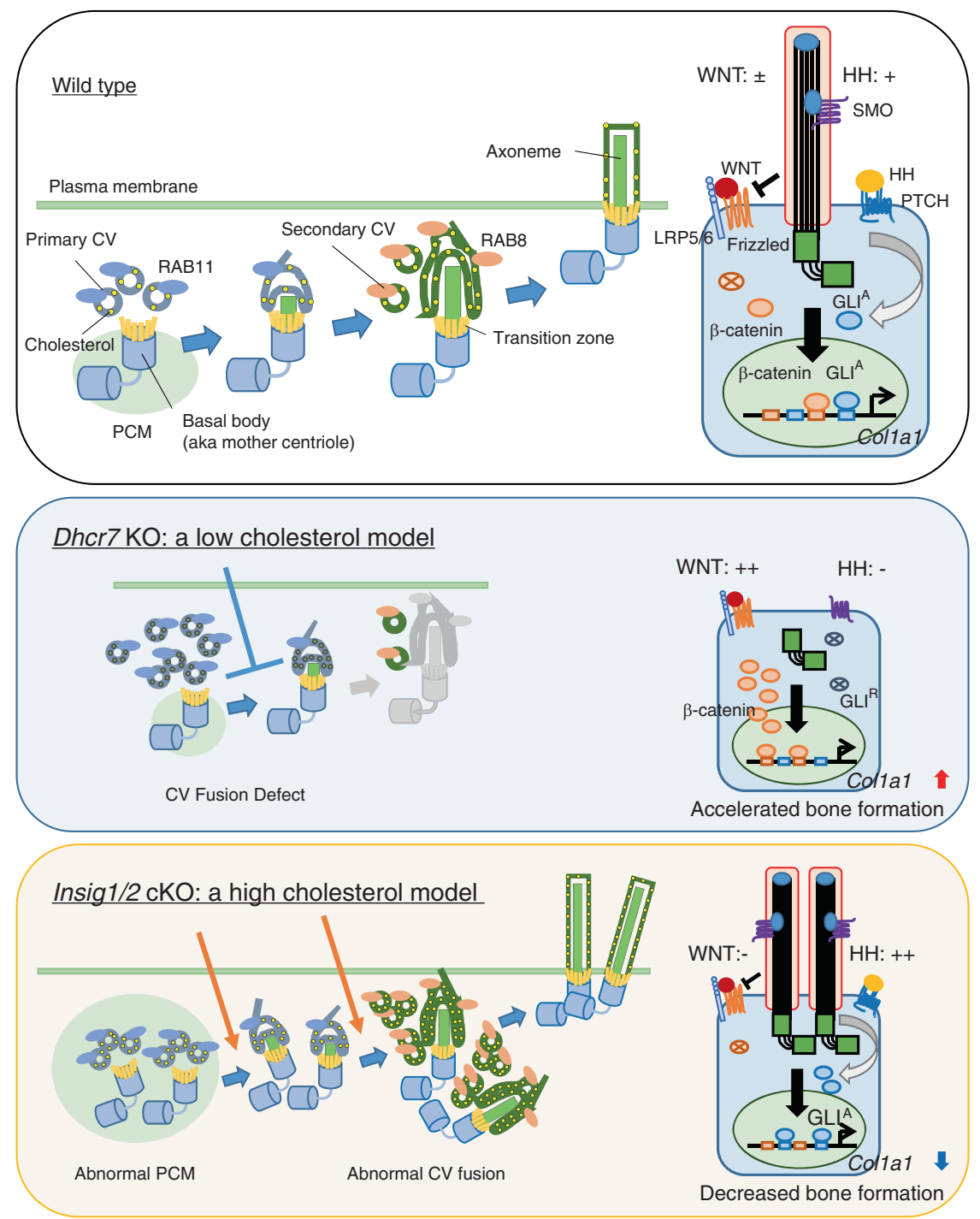

Fig. 9 Model of primary cilium formation altered in $\mathrm{Dhcr} 7^{-1-}$ and Insig1/2 mutant osteoblasts. Ciliogenesis starts with the interaction of the basal body (aka mother centriole) with primary ciliary vesicles (CVs), which can be labeled with RAB11, and then the axoneme grows within the ciliary membrane while fusing with secondary CVs, which can be labeled with RAB8. Eventually the elongated primary cilium fuses with the plasma membrane, allowing the distal part of the cilium to interact with the extracellular milieu. In Dhcr7 knockout (KO) osteoblasts, primary cilia were fewer and shorter than in controls. By contrast, Insig1/2 conditional KO (cKO) osteoblasts showed supernumerary and longer primary cilia compared to controls. Primary CVs labeled with RAB11 accumulated in the cells, and secondary CVs labeled with RAB8 failed to gather at cilium formation sites in Dhcr7 KO osteoblasts. Secondary CVs stained with RAB8 accumulated in Insig1/2 cKO osteoblasts, and the number of basal bodies was abnormally increased in Insig1/2 cKO osteoblasts. During osteogenesis, WT osteoblasts have a single primary cilium that suppresses WNT/ $\beta$-catenin signaling and activates $\mathrm{HH}$ signaling, which induces Col1a1 expression. In Dhcr7 KO osteoblasts, WNT/ $\beta$-catenin signaling is hyper-activated (WNT: ++ ) and $\mathrm{HH}$ signaling is compromised (HH: -), while in Insig1/2 cKO osteoblasts WNT/ $\beta$-catenin signaling is inhibited (WNT: - ) and $\mathrm{HH}$ signaling is hyper-activated $(\mathrm{HH}:++)$.

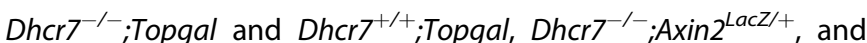
Dhcr ${ }^{+/+} ;$; Axin2 ${ }^{\text {LacZ/+ }}$ mice. Genotyping was performed using PCR primers, as previously described. ${ }^{12,22,85}$ Pregnant females were treated with simvastatin (S6196; Sigma-Aldrich) at a dose of $10 \mathrm{mg} / \mathrm{kg}^{-1}$ body weight (BW) from E12.5 to E18.5, or from day 7 to day 42 , administered by intraperitoneal injection.

Skeletal staining

The three-dimensional architecture of the skeleton was examined by modified whole-mount Alcian blue-Alizarin Red S staining, as previously described. ${ }^{88}$

\section{MicroCT}

MicroCT analysis was performed using a Scanco $\mu \mathrm{CT} 40$ unit in the microCT core facility at Baylor College of Medicine. The data were collected at a resolution of $20 \mu \mathrm{m}$. Three-dimensional reconstruction was performed using the BoneJ software.

\section{Histology}

$H \& E$, BrdU staining, von Kossa staining, LacZ staining, immunohistochemistry, and the TUNEL assay were performed as previously described. ${ }^{88,89}$ Antibodies used for immunohistochemistry were the anti-COL1A1 rabbit polyclonal (Abcam), anti-RUNX2 rabbit monoclonal (Cell Signaling Technology), anti-SP7 rabbit polyclonal (Abcam), anti-BrdU rat monoclonal (Abcam), and antiKi67 rabbit monoclonal (Abcam) antibodies (Supplementary Table S1). Click-iT ${ }^{\circledast}$ Plus TUNEL Assay with Alexa 594 (C10618, Molecular probes) was used to detect apoptotic cells, according to the manufacturer's instructions. Fluorescence images were obtained using a confocal microscope (Ti-C2, Nikon). 
12

Cell culture

Primary osteoblasts were obtained from newborn frontal bones. Briefly, the frontal bones were dissected at birth and incubated with $2 \mathrm{mg} \cdot \mathrm{mL}^{-1}$ collagenase II solution (Gibco) while shaking at $350 \mathrm{r} \cdot \mathrm{min}^{-1}$ at $37^{\circ} \mathrm{C}$ for $1 \mathrm{~h}$. Cells were resuspended in minimum essential medium alpha (MEM-a) supplemented with $10 \%$ fetal bovine serum (FBS), penicillin/streptomycin, and L-glutamine. For osteogenic differentiation, osteoblasts were cultured in 12-well plates, and osteogenic differentiation was induced with osteogenic induction medium (MEM-a supplemented with $100 \mu \mathrm{g} \cdot \mathrm{mL}^{-1}$ ascorbic acid, $5 \mathrm{mmol} \cdot \mathrm{L}^{-1}$ beta-glycerol phosphate, $10 \% \mathrm{FBS}$, penicillin/ streptomycin, and L-glutamine) for 28 days. To evaluate osteogenic differentiation, alkaline phosphatase staining, Alizarin Red staining, and von Kossa staining were performed. To induce ciliogenesis, osteoblasts were starved of serum for $24 \mathrm{~h}$, followed by immunofluorescent staining or collection of RNA and protein. To evaluate WNT signaling activity, osteoblasts were starved of serum for $24 \mathrm{~h}$ and then treated with either $20 \mathrm{mmol} \cdot \mathrm{L}^{-1}$ lithium chloride (LiCl) or $20 \mathrm{mmol} \cdot \mathrm{L}^{-1} \mathrm{NaCl}$ (a negative control) in a serum-free medium for $24 \mathrm{~h}$. For WNT3A treatment, WNT3A-conditioned medium and control medium were prepared from murine WNT3Aoverexpressing cells (L-Wnt3a cells: the American Type Culture Collection [ATCC]) and from control L-cells (ATCC), according to manufacturer's instructions, and used at $10 \%$ for each assay for $24 \mathrm{~h}$, respectively. For the cell proliferation assay, osteoblasts were plated onto 96-well plates at a density of 5000 cells per well and then counted by CCK8 (Dojindo Molecular Technologies) at 24, 48, and $72 \mathrm{~h}$. For the BrdU incorporation assay, osteoblasts were plated onto a 35-mm culture dish at a density of 10000 cells per dish for 2 days, and then $100 \mu \mathrm{g} \cdot \mathrm{mL}^{-1} \mathrm{BrdU}$ was added for $1 \mathrm{~h}$. To generate Insig1/2 CKO osteoblasts from Insig $1^{F / F} ;$ Insig2 ${ }^{-1-}$ osteoblasts, the Ad5-CMVLacZ-Cre virus (Vector Development Laboratory) was transduced at 300 multiplicity of infection (MOI) for 2 days. The Cre recombinase efficiency was evaluated with LacZ staining.

Organ cultures

Calvarial explants were dissected out from newborn $D h c r 7^{-1-}$ pups and incubated in BGjB medium (Gibco) supplemented with $10 \% \mathrm{FBS}, 0.1 \mathrm{mg} \cdot \mathrm{mL}^{-1}$ ascorbic acid, and penicillin/streptomycin, in a rotor incubator at $37^{\circ} \mathrm{C}$ for 3 days.

\section{Immunocytochemical analysis}

The ciliated osteoblasts plated onto ibiTreat 4-well $\mu$-slides (ibidi) were obtained through $24-h$ serum starvation. The antibodies used in immunocytochemistry were the anti- $\gamma$-tubulin mouse monoclonal (Sigma-Aldrich), anti-acetylated tubulin mouse monoclonal (Sigma-Aldrich), anti-RAB8 rabbit monoclonal (Cell signaling Technology), anti-RUNX2 rabbit monoclonal (Cell Signaling Technology), and anti-RAB11 rabbit polyclonal (Abcam) antibodies (Supplementary Table S1). Nuclei were counterstained with DAPI. The number and length of primary cilia were measured by NISElements (Nikon) under a confocal microscope (Ti-C2, Nikon).

Quantitative RT-PCR

Total messenger RNA was prepared as previously described. ${ }^{88}$ Quantitative RT-PCR was conducted using the following primers: Runx2, 5'-CGGACGAGGCAAGAGTTTCA-3' and 5'-GGATGAGGAAT GCGCCCTAA-3'; Alp, 5'-CTGAAGGCTCTCTTCACTCCAA-3' and 5'-AG GCGACAGGTGAAGAAACA-3'; Col1a1，5'-GAAGATGTAGGAGTCGA GGGAC-3' and 5'-CCTTGGAAACCTTGTGGACC-3'; Col1a2, 5'-CAAAG GCGTGAAAGGACACAG-3' and 5'-GCCAGTGAGCCCATTTGTTC-3'; Bglap, 5'-CCTAGCAGACACCATGAGGAC-3' and 5'-GTTGGCTTAG GGCAGCAC-3'; Sparc, 5'-GCCTACCACAAGGCAAGGAA-3' and 5'-CA GGTACCCCTGTCTCCTCC-3'; Sp7， 5'-GCCTGACTCCTTGGGACC-3' and 5'-TAGTGAGCTTCTTCCTCAAGCA-3'; Spp 1, 5'-AGTGACTGAT TCTGGCAGCTC-3' and 5'-ATTGCTTGGAAGAGTITCTTGCT-3'; Plk1, 5'- CCT TTG AGA CCT CGT GCC TA-3' and 5'- GGT TCT CCA CAC CTT TAT TGA GGA-3'; Plk4, 5'-AGACCGGCGGGAATाTाCA-3' and 5'-TA
AAGTCCTCGATCCTCTCCCC-3'; Sass6, 5'-GGAGAGGAGAGGGAGC GTTA-3' and 5'-CCTTGGAGTCTCTTTCGCGT-3'; Stil, 5'-TGCCTACG AGCCCAAATCAC-3' and 5'-TAGGCTTCACAGGCACACAC-3'; Axin2, 5'-GACGGACAGTAGCGTAGATGG-3' and 5'-CAGACTATGGCGGCT TTCCA-3'; Lef1, 5'-CGGGAAGAGCAGGCCAAATA-3' and 5'-CTGGG ACCTGTACCTGAAGTC-3'; Gli1, 5'-CACTGAGGACTTGTCCAGCTTG-3' and 5'-AGCTGGGCAGTITGAGACC-3'; Ptch1，5'-TAGCCCTGTGGT TCTTGTCC-3' and 5'-TGTGGTCATCCTGATTGCAT-3'; Wnt 1, 5'-ACTCATTGTCTGTGGCCCTG-3' and 5'-TATGTTCACGATGCCCCACC-3'; Wnt3a, 5'-GATCTGGTGGTCCTTGGCTG-3' and 5'- ACCCATCTATGCCATGCGAG-3'; Wnt7b, 5'-CACACTCTGGTCAACCTCCC-3' and 5'CAGCCTCTCGACTCCCTACT-3'; Wnt10b, 5'-TCTGGATCACTCCCTC CCTIT-3' and 5'- GTTACCACCTGGCGTCCC-3'; Wnt 16, 5'-TATGAGCTGAGTAGCGGCAC-3' and 5'- TCCAGCAGGTITTCACAGCA-3'; Fzd3, 5'-GCAGATAGGTGGGCACAGTT-3' and 5'- ATAGGGTGGAAGGGCT CCAT-3'; Fzd7, 5'-GGGGCGAGAGATGGTITGA-3' and 5'-AGGCTAC AGACAGAGCGGTA-3'; Fzd9, 5'-TCACCGTGTTCACCTTCCTG-3' and 5'- GCTTCTCCGTATTGGTGCCT-3' and Gapdh, 5'-AACTTTGGCATT GTGGAAGG-3' and 5'-ACACATTGGGGGTAGGAACA-3'.

\section{Immunoblotting}

Immunoblots were obtained as previously described. ${ }^{89}$ The antibodies used for immunoblotting were as follows: anticollagen type I rabbit polyclonal (Abcam), anti-INSIG1 rabbit polyclonal (Abcam), anti-INSIG2 rabbit polyclonal (Abcam), antiDHCR7 rabbit polyclonal (Abcam), anti-GLI1 rabbit polyclonal (Abcam), anti-non-phosphorylated (active) beta-catenin rabbit polyclonal (Cell Signaling Technology), anti-CREB rabbit polyclonal (Cell Signaling Technology), anti-SaK (aka PLK4) mouse monoclonal (Santa Cruz Biotechnology), and anti-GAPDH mouse monoclonal (Millipore) (Supplementary Table S1). Cell fractionation was performed using a NE-PER ${ }^{\text {TM }}$ nuclear and cytoplasmic extraction kit (Thermo Scientific).

Promoter analysis

The UCSC genome browser was used to obtain the genomic sequences of the Col1a1 murine gene (NC_000077.6) and the Plk4 murine gene (NC_000069.6), including the 5-kbp sequences upstream of the respective transcription start sites. The sequences were then mapped to seven additional mammalian genomes [human (Build 38), chimpanzee (Build 2.1.4), orangutan (Build 2.0.2), rhesus macaque (Build 1.0), rat (Build 5), dog (Build 3.1), and horse (Build equCab2)] with the BLAST tool, as previously described. ${ }^{90}$ Multiple alignments for these sequences were obtained using the Clustal Omega tool with default parameters and settings. LEF1 binding motifs (minimal core sites: $5^{\prime}-C A A A G-3^{\prime}$ and 5'-CTTG-3'; optimal sites: 5'-CTTGWW-3' and 5'-WWCAAAG$\left.3^{\prime}, W=A / T\right)$, the GLI-binding motif ( $\left.5^{\prime}-C A C C A C C C A-3\right),{ }^{91,92}$ and the SRE consensus sequence (5'-TCACNCCAC-3 $)^{93,94}$ were searched in the aligned DNA sequences, as previously described. ${ }^{90}$

\section{Chromatin immunoprecipitation assay}

At Day 3 of osteogenic differentiation, the osteoblast extracts were incubated with either active $\beta$-catenin (Cell signaling technology), GLI1 (Abcam), or normal rabbit IgG as a negative control (Santa Cruz Biotechnology) overnight at $4{ }^{\circ} \mathrm{C}$, followed by precipitation with magnetic beads. The osteoblast extracts were incubated with either mouse SREBP1 and SREBP2 antibodies (Santa Cruz Biotechnology) or normal mouse IgG (Santa Cruz Biotechnology) overnight at $4{ }^{\circ} \mathrm{C}$, followed by precipitation with magnetic beads. Washing and elution of the immune complexes, as well as precipitation of DNA, were performed according to standard procedures, as previously described. ${ }^{90}$ The putative LEF1/ $\beta$-catenin binding sites in the immune complexes were detected by PCR using the following primers: Colla1 site 1, 5'-AGCAGACGGGAGTITCTCCT-3' and 5'-GCAGCTGACTTCAGGGATGT-3' (-117 bp to + $93 \mathrm{bp}$ ); site 2, 5'-CAGGCTTCCTGCAACAAACT-3' and 5'AGGGGGTGCCTATCTGTTCT-3' (-985 bp to $-736 \mathrm{bp}$ ); site 3 , 5'- 
GTCCTTCCATTGCTGTCTCC- ${ }^{\prime}$ and 5'-CCATCCAAGATTCCATTGCT-3 (-1814 bp to -1569 bp); and site 4, 5'-TGGAGATTCTGGCTTTTGCT-3' and 5'-TGCAGCATGACAGAGAGAGG-3' (-2756 bp to $-2517 \mathrm{bp}$ ). The putative GLI-binding sites on the Col1a1 promotor were detected by the following primers: 5 '-CGGGACTITCTCCTCGGGG-3' ( -111 bp to -94 bp) and 5'-GGGGTTAGCTTCGGCTCA-3' ( -59 bp to $-42 \mathrm{bp})$. The putative SREs on the Plk4 promotor in the immune complexes were detected by PCR using the following primers: site 1, 5'-AAACCCACTTCCGGCCTAGA-3' (-322 bp to -303 bp) and 5'TGAAAAATTCCCGCCGGTCT-3' (-210 bp to $-191 \mathrm{bp})$; and site 2, 5'GCTTGCAGGATAACGTGTTCATT-3' (-1402 bp to $-1380 \mathrm{bp})$ and 5'AATAAGAGGAATAGGCTAGCGGG-3' (-1275 bp to $-1262 \mathrm{bp})$. The position of the PCR fragments corresponds to NCBI mouse genome Build 38 (mm10).

\section{Statistics}

The two-tailed student's $t$-test was applied for statistical analysis. A $P$-value $<0.05$ was considered statistically significant. For all graphs, data are represented as mean \pm standard deviation.

Study approval

All animal experiments were reviewed and approved by the Animal Welfare Committee and the Institutional Animal Care and Use Committee of UTHealth.

\section{ACKNOWLEDGEMENTS}

We thank Dr. Yoshihiro Komatsu for fruitful discussions. This study was supported by grants from the National Institute of Dental and Craniofacial Research, NIH (DE024759, DE026208, DE026509, and DE026767), to J.I. and UTHealth School of Dentistry faculty funds to J.I.

\section{AUTHOR CONTRIBUTIONS}

A.S., K.O., H.Y., J.S. and J.I. performed the experiments. A.S. and J.I. wrote the manuscript. C.A.W. and F.D.P. provided the mice analyzed in this study and revised the manuscript. All authors read and approved the final manuscript.

\section{ADDITIONAL INFORMATION}

The online version of this article (https://doi.org/10.1038/s41413-019-0078-3) contains supplementary material, which is available to authorized users.

Competing interests: The authors declare no competing interests.

\section{REFERENCES}

1. Porter, F. D. \& Herman, G. E. Malformation syndromes caused by disorders of cholesterol synthesis. J. Lipid Res. 52, 6-34 (2011).

2. Iwata, J. et al. Modulation of lipid metabolic defects rescues cleft palate in Tgfbr2 mutant mice. Hum. Mol. Genet. 23, 182-193 (2014).

3. Engelking, L. J. et al. Severe facial clefting in Insig-deficient mouse embryos caused by sterol accumulation and reversed by lovastatin. J. Clin. Invest. 116, 2356-2365 (2006).

4. Rossi, M. et al. Clinical phenotype of lathosterolosis. Am. J. Med. Genet. A 143A, 2371-2381 (2007).

5. Porter, F. D. Smith-Lemli-Opitz syndrome: pathogenesis, diagnosis and management. Eur. J. Hum. Genet. 16, 535-541 (2008).

6. Edison, R. J. et al. Adverse birth outcome among mothers with low serum cholesterol. Pediatrics 120, 723-733 (2007).

7. Maymunah, A. O., Kehinde, O., Abidoye, G. \& Oluwatosin, A. Hypercholesterolaemia in pregnancy as a predictor of adverse pregnancy outcome. Afr. Health Sci. 14, 967-973 (2014).

8. Battaile, K. P. \& Steiner, R. D. Smith-Lemli-Opitz syndrome: the first malformation syndrome associated with defective cholesterol synthesis. Mol. Genet. Metab. 71, 154-162 (2000)

9. Fitzky, B. U. et al. Mutations in the Delta7-sterol reductase gene in patients with the Smith-Lemli-Opitz syndrome. Proc. Natl Acad. Sci. USA 95, 8181-8186 (1998).

10. Porter, F. D. RSH/Smith-Lemli-Opitz syndrome: a multiple congenital anomaly/ mental retardation syndrome due to an inborn error of cholesterol biosynthesis. Mol. Genet. Metab. 71, 163-174 (2000).
11. Fitzky, B. U. et al. 7-Dehydrocholesterol-dependent proteolysis of HMG-CoA reductase suppresses sterol biosynthesis in a mouse model of Smith-Lemli-Opitz/ RSH syndrome. J. Clin. Invest. 108, 905-915 (2001).

12. Engelking, L. J. et al. Schoenheimer effect explained-feedback regulation of cholesterol synthesis in mice mediated by Insig proteins. J. Clin. Invest. 115 2489-2498 (2005).

13. Dong, X. Y., Tang, S. Q. \& Chen, J. D. Dual functions of Insig proteins in cholesterol homeostasis. Lipids Health Dis. 11, 173 (2012).

14. Emmer, B. T., Maric, D. \& Engman, D. M. Molecular mechanisms of protein and lipid targeting to ciliary membranes. J. Cell Sci. 123, 529-536 (2010).

15. Senin, I. I. et al. Recoverin and rhodopsin kinase activity in detergent-resistant membrane rafts from rod outer segments. J. Biol. Chem. 279, 48647-48653 (2004).

16. Iomini, C., Li, L., Mo, W., Dutcher, S. K. \& Piperno, G. Two flagellar genes, AGG2 and AGG3, mediate orientation to light in Chlamydomonas. Curr. Biol.: $C B$ 16, 1147-1153 (2006).

17. Travis, A. J. et al. Expression and localization of caveolin-1, and the presence of membrane rafts, in mouse and Guinea pig spermatozoa. Developmental Biol. 240, 599-610 (2001).

18. Tull, D. et al. SMP-1, a member of a new family of small myristoylated proteins in kinetoplastid parasites, is targeted to the flagellum membrane in Leishmania. Mol. Biol. Cell 15, 4775-4786 (2004).

19. Chang, C. F., Schock, E. N., Attia, A. C., Stottmann, R. W. \& Brugmann, S. A. The ciliary baton: orchestrating neural crest cell development. Curr. Top. Dev. Biol. 111, 97-134 (2015).

20. Nachury, M. V., Seeley, E. S. \& Jin, H. Trafficking to the ciliary membrane: how to get across the periciliary diffusion barrier? Annu Rev. Cell Dev. Biol. 26, 59-87 (2010).

21. Goetz, S. C. \& Anderson, K. V. The primary cilium: a signalling centre during vertebrate development. Nat. Rev. Genet. 11, 331-344 (2010).

22. Lewis, A. E., Vasudevan, H. N., O'Neill, A. K., Soriano, P. \& Bush, J. O. The widely used Wnt1-Cre transgene causes developmental phenotypes by ectopic activation of Wnt signaling. Dev. Biol. 379, 229-234 (2013).

23. Chai, Y. et al. Fate of the mammalian cranial neural crest during tooth and mandibular morphogenesis. Development 127, 1671-1679 (2000).

24. Wilkie, A. O. Craniosynostosis: genes and mechanisms. Hum. Mol. Genet. 6, 1647-1656 (1997).

25. Knodler, A. et al. Coordination of Rab8 and Rab11 in primary ciliogenesis. Proc. Natl Acad. Sci. USA 107, 6346-6351 (2010).

26. Westlake, C. J. et al. Primary cilia membrane assembly is initiated by Rab11 and transport protein particle II (TRAPPII) complex-dependent trafficking of Rabin8 to the centrosome. Proc. Natl Acad. Sci. USA 108, 2759-2764 (2011).

27. Pedersen, L. B., Veland, I. R., Schroder, J. M. \& Christensen, S. T. Assembly of primary cilia. Dev. Dyn. 237, 1993-2006 (2008).

28. Arquint, C., Sonnen, K. F., Stierhof, Y. D. \& Nigg, E. A. Cell-cycle-regulated expression of STIL controls centriole number in human cells. J. Cell Sci. 125 1342-1352 (2012).

29. Ko, M. A. et al. Plk4 haploinsufficiency causes mitotic infidelity and carcinogenesis. Nat. Genet. 37, 883-888 (2005).

30. Kong, D. et al. Centriole maturation requires regulated Plk1 activity during two consecutive cell cycles. J. Cell Biol. 206, 855-865 (2014).

31. Pearson, C. G. \& Winey, M. Plk4/SAK/ZYG-1 in the regulation of centriole duplication. F1000 Biol. Rep. 2, 58 (2010).

32. Peel, N., Stevens, N. R., Basto, R. \& Raff, J. W. Overexpressing centriole-replication proteins in vivo induces centriole overduplication and de novo formation. Curr. Biol. 17, 834-843 (2007).

33. Strnad, P. et al. Regulated HsSAS-6 levels ensure formation of a single procentriole per centriole during the centrosome duplication cycle. Dev. Cell 13, 203-213 (2007).

34. Tsou, M. F. et al. Polo kinase and separase regulate the mitotic licensing of centriole duplication in human cells. Dev. Cell 17, 344-354 (2009).

35. Vulprecht, J. et al. STIL is required for centriole duplication in human cells. J. Cell Sci. 125, 1353-1362 (2012).

36. Day, T. F. \& Yang, Y. Wnt and hedgehog signaling pathways in bone development. J. Bone Jt. Surg. Am. 90(Suppl 1), 19-24 (2008)

37. Gaur, T. et al. Canonical WNT signaling promotes osteogenesis by directly stimulating Runx2 gene expression. J. Biol. Chem. 280, 33132-33140 (2005).

38. Yuan, X. \& Yang, S. Primary cilia and intraflagellar transport proteins in bone and cartilage. J. Dent. Res. 95, 1341-1349 (2016).

39. Bai, C. B., Auerbach, W., Lee, J. S., Stephen, D. \& Joyner, A. L. Gli2, but not Gli1, is required for initial Shh signaling and ectopic activation of the Shh pathway. Development 129, 4753-4761 (2002).

40. Goodrich, L. V., Johnson, R. L., Milenkovic, L., McMahon, J. A. \& Scott, M. P. Conservation of the hedgehog/patched signaling pathway from flies to mice: induction of a mouse patched gene by Hedgehog. Genes Dev. 10, 301-312 (1996). 
41. Ingham, P. W. Localized hedgehog activity controls spatial limits of wingless transcription in the Drosophila embryo. Nature 366, 560-562 (1993).

42. Marigo, V., Johnson, R. L., Vortkamp, A. \& Tabin, C. J. Sonic hedgehog differentially regulates expression of GLI and GLI3 during limb development. Dev. Biol. 180, 273-283 (1996).

43. Platt, K. A., Michaud, J. \& Joyner, A. L. Expression of the mouse Gli and Ptc genes is adjacent to embryonic sources of hedgehog signals suggesting a conservation of pathways between flies and mice. Mech. Dev. 62, 121-135 (1997).

44. Aza-Blanc, P., Ramirez-Weber, F. A., Laget, M. P., Schwartz, C. \& Kornberg, T. B. Proteolysis that is inhibited by hedgehog targets Cubitus interruptus protein to the nucleus and converts it to a repressor. Cell 89, 1043-1053 (1997).

45. Aza-Blanc, P. \& Lin, H. Y., Ruiz i Altaba, A. \& Kornberg, T. B. Expression of the vertebrate Gli proteins in Drosophila reveals a distribution of activator and repressor activities. Development 127, 4293-4301 (2000).

46. Hammond, N. L., Brookes, K. J. \& Dixon, M. J. Ectopic hedgehog signaling causes cleft palate and defective osteogenesis. J. Dent. Res., 22034518785336, https:// doi.org/10.1177/0022034518785336 (2018).

47. St-Jacques, B., Hammerschmidt, M. \& McMahon, A. P. Indian hedgehog signaling regulates proliferation and differentiation of chondrocytes and is essential for bone formation. Genes Dev. 13, 2072-2086 (1999).

48. Lenton, $\mathrm{K}$. et al. Indian hedgehog positively regulates calvarial ossification and modulates bone morphogenetic protein signaling. Genesis 49, 784-796 (2011).

49. McMurray, R. J., Wann, A. K., Thompson, C. L., Connelly, J. T. \& Knight, M. M. Surface topography regulates wnt signaling through control of primary cilia structure in mesenchymal stem cells. Sci. Rep. 3, 3545 (2013).

50. $\mathrm{Yu}, \mathrm{H}$. M. et al. The role of Axin2 in calvarial morphogenesis and craniosynostosis. Development 132, 1995-2005 (2005).

51. Lancaster, M. A., Schroth, J. \& Gleeson, J. G. Subcellular spatial regulation of canonical Wnt signalling at the primary cilium. Nat. Cell Biol. 13, 700-707 (2011).

52. Baron, R. \& Kneissel, M. WNT signaling in bone homeostasis and disease: from human mutations to treatments. Nat. Med. 19, 179-192 (2013).

53. Ali, S. A. et al. Regulation of cholesterol homeostasis by hedgehog signaling in osteoarthritic cartilage. Arthritis Rheumatol. 68, 127-137 (2016).

54. Tsushima, H. et al. Intracellular biosynthesis of lipids and cholesterol by Scap and Insig in mesenchymal cells regulates long bone growth and chondrocyte homeostasis. Development 145, https://doi.org/10.1242/dev.162396 (2018).

55. Holick, M. F. Vitamin D: a millenium perspective. J. Cell Biochem. 88, 296-307 (2003).

56. Morris, J. G., Earle, K. E. \& Anderson, P. A. Plasma 25-hydroxyvitamin D in growing kittens is related to dietary intake of cholecalciferol. J. Nutr. 129, 909-912 (1999).

57. Prabhu, A. V., Luu, W., Sharpe, L. J. \& Brown, A. J. Cholesterol-mediated degradation of 7-dehydrocholesterol reductase switches the balance from cholesterol to vitamin D synthesis. J. Biol. Chem. 291, 8363-8373 (2016).

58. Bonjour, J. P. et al. The increase in skin 7-dehydrocholesterol induced by an hypocholesterolemic agent is associated with elevated 25-hydroxyvitamin D3 plasma level. Pflug. Arch. 410, 165-168 (1987).

59. Movassaghi, M., Bianconi, S., Feinn, R., Wassif, C. A. \& Porter, F. D. Vitamin D levels in Smith-Lemli-Opitz syndrome. Am. J. Med Genet. A 173, 2577-2583 (2017).

60. Novakovic, B. et al. Maternal vitamin D predominates over genetic factors in determining neonatal circulating vitamin D concentrations. Am. J. Clin. Nutr. 96, 188-195 (2012).

61. Stordal, K. et al. Fetal and maternal genetic variants influencing neonatal vitamin D status. J. Clin. Endocrinol. Metab. 102, 4072-4079 (2017).

62. Kelley, R. I. Inborn errors of cholesterol biosynthesis. Adv. Pediatr. 47, 1-53 (2000).

63. Lindegaard, M. L. et al. Characterization of placental cholesterol transport: ABCA1 is a potential target for in utero therapy of Smith-Lemli-Opitz syndrome. Hum. Mol. Genet. 17, 3806-3813 (2008).

64. Tint, G. S., Yu, H., Shang, Q., Xu, G. \& Patel, S. B. The use of the Dhcr7 knockout mouse to accurately determine the origin of fetal sterols. J. Lipid Res. 47, 1535-1541 (2006)

65. Hoey, D. A., Chen, J. C. \& Jacobs, C. R. The primary cilium as a novel extracellular sensor in bone. Front. Endocrinol. 3, 75 (2012).

66. Anderson, C. T. et al. Primary cilia: cellular sensors for the skeleton. Anat. Rec. 291, 1074-1078 (2008).

67. Qiu, N. et al. Disruption of Kif3a in osteoblasts results in defective bone formation and osteopenia. J. Cell Sci. 125, 1945-1957 (2012).

68. Lehti, M. S. et al. Cilia-related protein SPEF2 regulates osteoblast differentiation. Sci. Rep. 8, 859 (2018)

69. Cooper, M. K. et al. A defective response to Hedgehog signaling in disorders of cholesterol biosynthesis. Nat. Genet. 33, 508-513 (2003).

70. Blassberg, R., Macrae, J. I., Briscoe, J. \& Jacob, J. Reduced cholesterol levels impair Smoothened activation in Smith-Lemli-Opitz syndrome. Hum. Mol. Genet. 25, 693-705 (2016).

71. Blassberg, R. \& Jacob, J. Lipid metabolism fattens up hedgehog signaling. BMC Biol. 15, 95 (2017).
72. Abzhanov, A., Rodda, S. J., McMahon, A. P. \& Tabin, C. J. Regulation of skeletogenic differentiation in cranial dermal bone. Development 134, 3133-3144 (2007).

73. Jacob, S., Wu, C., Freeman, T. A., Koyama, E. \& Kirschner, R. E. Expression of Indian Hedgehog, BMP-4 and Noggin in craniosynostosis induced by fetal constraint. Ann. Plast. Surg. 58, 215-221 (2007).

74. Veistinen, L. K. et al. Regulation of calvarial osteogenesis by concomitant derepression of GLI3 and activation of IHH targets. Front Physiol. 8, 1036 (2017).

75. Jeong, J., Mao, J., Tenzen, T., Kottmann, A. H. \& McMahon, A. P. Hedgehog signaling in the neural crest cells regulates the patterning and growth of facial primordia. Genes Dev. 18, 937-951 (2004).

76. Lan, Y. \& Jiang, R. Sonic hedgehog signaling regulates reciprocal epithelialmesenchymal interactions controlling palatal outgrowth. Development 136, 1387-1396 (2009).

77. Rice, R. et al. Disruption of Fgf10/Fgfr2b-coordinated epithelial-mesenchymal interactions causes cleft palate. J. Clin. Invest. 113, 1692-1700 (2004).

78. Cobourne, M. T. et al. Sonic hedgehog signalling inhibits palatogenesis and arrests tooth development in a mouse model of the nevoid basal cell carcinoma syndrome. Dev. Biol. 331, 38-49 (2009).

79. Tu, X. et al. Osteocytes mediate the anabolic actions of canonical Wnt/betacatenin signaling in bone. Proc. Natl Acad. Sci. USA 112, E478-486, (2015).

80. Chen, S. et al. Adverse effects of osteocytic constitutive activation of ss-catenin on bone strength and bone growth. J. Bone Miner. Res.: Off. J. Am. Soc. Bone Miner. Res. 30, 1184-1194, (2015).

81. Jia, M. et al. Effects of constitutive beta-catenin activation on vertebral bone growth and remodeling at different postnatal stages in mice. PLOS ONE 8, e74093, (2013).

82. Day, T. F., Guo, X., Garrett-Beal, L. \& Yang, Y. Wnt/beta-catenin signaling in mesenchymal progenitors controls osteoblast and chondrocyte differentiation during vertebrate skeletogenesis. Dev. Cell 8, 739-750, (2005).

83. Corbit, K. C. et al. Kif3a constrains beta-catenin-dependent Wnt signalling through dual ciliary and non-ciliary mechanisms. Nat. Cell Biol. 10, 70-76 (2008).

84. Brugmann, S. A. et al. A primary cilia-dependent etiology for midline facial disorders. Hum. Mol. Genet. 19, 1577-1592 (2010).

85. Correa-Cerro, L. S. et al. Development and characterization of a hypomorphic Smith-Lemli-Opitz syndrome mouse model and efficacy of simvastatin therapy. Hum. Mol. Genet. 15, 839-851 (2006).

86. DasGupta, R. \& Fuchs, E. Multiple roles for activated LEF/TCF transcription complexes during hair follicle development and differentiation. Development 126, 4557-4568 (1999).

87. Lustig, B. et al. Negative feedback loop of Wnt signaling through upregulation of conductin/axin2 in colorectal and liver tumors. Mol. Cell. Biol. 22, 1184-1193 (2002).

88. Iwata, J. et al. Modulation of noncanonical TGF-beta signaling prevents cleft palate in Tgfbr2 mutant mice. J. Clin. Invest. 122, 873-885, (2012).

89. Iwata, J. et al. Transforming growth factor-beta regulates basal transcriptional regulatory machinery to control cell proliferation and differentiation in cranial neural crest-derived osteoprogenitor cells. J. Biol. Chem. 285, 4975-4982 (2010).

90. Suzuki, A., Pelikan, R. C. \& Iwata, J. WNT/beta-catenin signaling regulates multiple steps of myogenesis by regulating step-specific targets. Mol. Cell. Biol. 35, 1763-1776 (2015).

91. Kinzler, K. W. \& Vogelstein, B. The GLI gene encodes a nuclear protein which binds specific sequences in the human genome. Mol. Cell. Biol. 10, 634-642 (1990).

92. Winklmayr, M. et al. Non-consensus GLI binding sites in Hedgehog target gene regulation. BMC Mol. Biol. 11, 2 (2010).

93. Shimano, H. Sterol regulatory element-binding proteins (SREBPs): transcriptional regulators of lipid synthetic genes. Prog. Lipid Res. 40, 439-452 (2001).

94. Edwards, P. A., Tabor, D., Kast, H. R. \& Venkateswaran, A. Regulation of gene expression by SREBP and SCAP. Biochimica et. Biophysica Acta 1529, 103-113 (2000).

Open Access This article is licensed under a Creative Commons Attribution 4.0 International License, which permits use, sharing, adaptation, distribution and reproduction in any medium or format, as long as you give appropriate credit to the original author(s) and the source, provide a link to the Creative Commons license, and indicate if changes were made. The images or other third party material in this article are included in the article's Creative Commons license, unless indicated otherwise in a credit line to the material. If material is not included in the article's Creative Commons license and your intended use is not permitted by statutory regulation or exceeds the permitted use, you will need to obtain permission directly from the copyright holder. To view a copy of this license, visit http://creativecommons. org/licenses/by/4.0/.

(c) The Author(s) 2020 